\title{
Linearly implicit Rosenbrock-type Runge-Kutta schemes applied to the Discontinuous Galerkin solution of compressible and incompressible unsteady flows
}

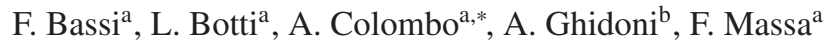 \\ ${ }^{a}$ Università degli Studi di Bergamo, Dipartimento di Ingegneria e Scienze Applicate \\ ${ }^{b}$ Università degli Studi di Brescia, Dipartimento di Ingegneria Meccanica e Industriale
}

\begin{abstract}
In this work we investigate the use of linearly implicit Rosenbrock-type Runge-Kutta schemes to integrate in time high-order Discontinuous Galerkin space discretizations of the Navier-Stokes equations. The final goal of this activity is the application of such schemes to the high-order accurate, both in space and time, simulation of turbulent flows. Besides being able to overcome the severe time step restriction of explicit schemes, Rosenbrock schemes have the attractive feature of requiring just one Jacobian matrix evaluation per time step, thus reducing the overall computational effort. Several high-order (up to sixth order) Rosenbrock schemes available in the literature have been implemented and evaluated on benchmark test cases of both compressible and incompressible flows. For the sake of completeness, the sets of coefficients of the schemes here considered have been reported in an Appendix to the paper. An implementation of Rosenbrock schemes for systems of equations with a solution dependent block diagonal matrix multiplying the time derivative is here proposed and described in detail. This can occur, for example, if sets of working variables different from the conservative ones are used in the compressible NavierStokes equations. In particular, we have found useful to employ primitive variables based on the logarithms of pressure and temperature in order to ensure the positivity of all thermodynamic variables at the discrete level. The best performing Rosenbrock scheme resulting from our analysis has then been applied to the Implicit Large Eddy Simulation of the transitional flow around the Selig-Donovan SD7003 airfoil.
\end{abstract}

Keywords: Discontinuous Galerkin discretization, high-order accurate time integration, linearly implicit Rosenbrock-type Runge-Kutta schemes, compressible and incompressible flows

\section{Introduction}

In recent years the application of the Discontinuous Galerkin (DG) method to the high-fidelity simulation of turbulent flows has become increasingly popular. Thanks to its favourable dissipation and dispersion properties, the DG method proved to be very well suited for the Direct Numerical Simulation (DNS), [1, 2, 3], and the Large Eddy Simulation (LES), [4, 5], of turbulent flows. The DG method has also been recently applied by Bassi et al., [6], to hybrid RANS-LES

*Corresponding author: alessandro.colombo@ unibg.it, Tel +39 0352052150, fax +39 0352052077 Preprint submitted to Computers and Fluids 
approaches, [7, 8, 9], in an attempt to overcome the poor predictive capabilities of the Reynoldsaveraged Navier-Stokes (RANS) equations with first-moment closures in case of particular flow conditions, e.g., massively separated flows.

Advanced approaches to the simulation of turbulent flows require accurate long term time integration of unsteady flows characterized by a wide range of temporal scales. In this context, the locality and flexibility of the DG space discretization turns out very useful both for explicit and implicit time integration approaches. In general, explicit schemes can be of high accuracy and simple to implement, but a search for high levels of efficiency can also lead to very sophisticated algorithms, $[10,11]$. On the other hand, implicit methods require in general to form the Jacobian matrix of residuals, a task that leads to high memory usage, but that can be quite systematically accomplished in the framework of the DG space discretization. In an attempt to combine the advantages of explicit and implicit approaches, also implicit-explicit (IMEX) Runge-Kutta schemes have been recently applied to LES of turbulent flows by Persson, [12].

Multistep Backward Differentiation Formulae (BDF), [13], are often used as implicit time integrators, due to ease of implementation and robustness. However, since BDF schemes are $A$-stable only up to second order, they might not always be the best choice for matching very high-order space discretizations, which are in increasingly widespread use for turbulent flow simulations. Several high-order implicit time integrators, relying on multistage and multistep schemes, are already available. For example, Explicit Singly Diagonally Implicit Runge-Kutta (ESDIRK) schemes, [14, 15], are $A$-stable up to order five, Modified Extended BDF (MEBDF), [16, 17, 18], are $A$-stable up to order four, and Two Implicit Advanced Step-point (TIAS) schemes, [19, 20], are $A$-stable up to order six. At each time step, all these schemes require to solve several nonlinear systems of equations, a task that can be efficiently performed, for example, by means of the (quasi-)Newton method.

In the large family of implicit Runge-Kutta methods, the class of linearly implicit Rosenbrocktype Runge-Kutta schemes, [21], is of special interest because such methods, being linearly implicit, require to solve only linear systems in the stages within each time step, i.e., the Jacobian matrix needs to be assembled and factored only once per time step. The performance of all the aforementioned time integration schemes have been recently investigated, and, according to the numerical comparison presented in [22], Rosenbrock schemes turned out to be an appealing choice both in terms accuracy and efficiency. In this paper we focus on the implementation and assessment of Rosenbrock schemes as time integrators for the high-order DG space discretization of the compressible and incompressible Navier-Stokes equations. In particular, we present an implementation of Rosenbrock schemes for systems of equations with a solution dependent block diagonal matrix multiplying the time derivative. For compressible flows, this situation occurs if the polynomial approximation of the unknowns in the governing equations is not applied to the set of conservative variables but to a different set, like, for example, the primitive variables based on pressure and temperature. Among other reasons for working with a polynomial approximation of primitive variables, here we want to emphasize the possibility of ensuring the positivity of all thermodynamic variables at the discrete level by using a polynomial approximation for the logarithms of pressure and temperature, [6].

In this work we have carried out a comparative assessment, in terms of accuracy and performance, of several (up to sixth order) Rosenbrock schemes, [23, 24, 25, 26, 27, 28, 29], on two benchmark testcases, i.e., the convection of a compressible isentropic vortex and a travelling waves problem for incompressible flow. In particular, the following aspects have been investigated in detail: (i) temporal order of convergence of the schemes; (ii) optimal choice of parameters of the iterative linear solver; (iii) computational efficiency of the schemes to obtain a 
given accuracy. Then, guided by the results of the above comparison, one of the best performing high-order Rosenbrock schemes has been applied to the high-order DG Implicit Large Eddy Simulation (ILES) of the transitional flow around the Selig-Donovan (SD) 7003 airfoil. This test case has been considered by several other authors using different numerical methods and LES models, e.g. [5, 30, 31, 32, 33, 34, 35].

The rest of the paper is organized as follows. A quick overview of the governing equations is given in Sec. 2. In Sec. 3, devoted to space and time discretization, the use of different sets of working variables in the DG discretized equations is considered, and the related implementation of Rosenbrock schemes is devised. Sec. 4 deals with the accuracy and performance assessment of the time integration schemes and presents the results of an ILES simulation. The conclusions are given in Sec. 5 and all the sets of coefficients of the Rosenbrock schemes considered in this paper are reported in Appendix B.

\section{Governing Equations}

Using Einstein notation, the Navier-Stokes equations for compressible flows read

$$
\begin{aligned}
\frac{\partial \rho}{\partial t}+\frac{\partial}{\partial x_{j}}\left(\rho u_{j}\right) & =0, \\
\frac{\partial}{\partial t}\left(\rho u_{i}\right)+\frac{\partial}{\partial x_{j}}\left(\rho u_{j} u_{i}\right) & =-\frac{\partial p}{\partial x_{i}}+\frac{\partial \tau_{j i}}{\partial x_{j}}, \\
\frac{\partial}{\partial t}(\rho E)+\frac{\partial}{\partial x_{j}}\left(\rho u_{j} H\right) & =\frac{\partial}{\partial x_{j}}\left[u_{i} \tau_{i j}-q_{j}\right],
\end{aligned}
$$

where $E$ and $H$ are total energy and total enthalpy, respectively. The pressure, stress tensor and heat flux vector are given by

$$
\begin{gathered}
p=(\gamma-1) \rho\left(E-u_{k} u_{k} / 2\right), \\
\tau_{i j}=2 \mu\left[S_{i j}-\frac{1}{3} \frac{\partial u_{k}}{\partial x_{k}} \delta_{i j}\right], \\
q_{j}=-\frac{\mu}{\operatorname{Pr}} \frac{\partial h}{\partial x_{j}},
\end{gathered}
$$

where $\gamma$ is the ratio of gas specific heats, $S_{i j}$ is the mean strain-rate tensor

$$
S_{i j}=\frac{1}{2}\left(\frac{\partial u_{i}}{\partial x_{j}}+\frac{\partial u_{j}}{\partial x_{i}}\right),
$$

and Pr is the molecular Prandtl number.

For incompressible flows, we consider the set of governing equations

$$
\begin{gathered}
\frac{\partial u_{j}}{\partial x_{j}}=0 \\
\frac{\partial u_{i}}{\partial t}+\frac{\partial}{\partial x_{j}}\left(u_{j} u_{i}\right)=-\frac{\partial p}{\partial x_{i}}+\frac{\partial \tau_{j i}}{\partial x_{j}},
\end{gathered}
$$

where the density has been assumed to be uniform and equal to one. The stress tensor $\tau_{i j}$ is again computed from Eq. (5), assuming a constant $\mu$ and keeping the velocity divergence in this equation. 


\section{Space and time discretization}

\subsection{The DG discrete setting}

Let $\mathcal{T}_{h}=\{K\}$ denote a mesh of the domain $\Omega \in \mathbb{R}^{d}, d \in\{2,3\}$ consisting of non-overlapping arbitrarily shaped elements $K$ such that

$$
\bar{\Omega}_{h}=\bigcup_{K \in \mathcal{T}_{h}} \bar{K}
$$

Following the idea to define discrete polynomial spaces in physical coordinates, see, e.g., [36, $37,38,39,40,41]$, we consider DG approximations based on the space

$$
\mathbb{P}_{d}^{k}\left(\mathcal{T}_{h}\right) \stackrel{\text { def }}{=}\left\{v_{h} \in L^{2}(\Omega) \mid v_{h \mid K} \in \mathbb{P}_{d}^{k}(K), \forall K \in \mathcal{T}_{h}\right\},
$$

where $k$ is a non-negative integer and $\mathbb{P}_{d}^{k}(K)$ denotes the restriction to $K$ of the polynomial functions of $d$ variables and total degree $\leq k$. To build a satisfactory basis for the space (10) we rely on the procedure presented in [42], see also [43, 44], allowing to obtain orthonormal and hierarchical basis functions by means of the modified Gram-Schmidt (MGS) algorithm. The starting set of basis functions for the MGS algorithm are the monomials defined over each elementary space $\mathbb{P}_{d}^{k}(K), K \in \mathcal{T}_{h}$, in a reference frame relocated in the element barycenter and aligned with the principal axes of inertia of $K$. For the sake of presenting the DG discretization, we introduce the set $\mathcal{F}_{h}$ of the mesh faces $\mathcal{F}_{h} \stackrel{\text { def }}{=} \mathcal{F}_{h}^{i} \cup \mathcal{F}_{h}^{b}$, where $\mathcal{F}_{h}^{b}$ collects the faces located on the boundary of $\Omega_{h}$ and for any $F \in \mathcal{F}_{h}^{i}$ there exist two elements $K^{+}, K^{-} \in \mathcal{T}_{h}$ such that $F \in \partial K^{+} \cap \partial K^{-}$. Moreover, for all $F \in \mathcal{F}_{h}^{b}, \mathbf{n}_{F}$ denotes the unit outward normal to $\Omega_{h}$, whereas, for all $F \in \mathcal{F}_{h}^{i}, \mathbf{n}_{F}^{-}$ and $\mathbf{n}_{F}^{+}$are unit outward normals pointing to $K^{+}$and $K^{-}$, respectively.

Since a function $v_{h} \in \mathbb{P}_{d}^{k}\left(\mathcal{T}_{h}\right)$ is double valued over an internal face $F \in \mathcal{F}_{h}^{i}$, we introduce the jump $\llbracket \cdot \rrbracket$ and average $\{\cdot\}$ trace operators, that is

$$
\llbracket v_{h} \rrbracket \stackrel{\text { def }}{=} v_{h \mid K^{+}} \mathbf{n}_{F}^{+}+v_{h \mid K^{-}} \mathbf{n}_{F}^{-}, \quad\left\{v_{h}\right\} \stackrel{\text { def }}{=} \frac{v_{h \mid K^{+}}+v_{h \mid K^{-}}}{2},
$$

and consider them to act componentwise when applied to vector functions. Finally, the DG discretization of second-order viscous terms employs the lifting operators $\mathbf{r}_{F}$ and $\mathbf{r}$. For all $F \in \mathcal{F}_{h}$, we define the local lifting operator $\mathbf{r}_{F}:\left[L^{2}(F)\right]^{d} \rightarrow\left[\mathbb{P}_{d}^{k}\left(\mathcal{T}_{h}\right)\right]^{d}$, such that, for all $\mathbf{v} \in\left[L^{2}(F)\right]^{d}$,

$$
\int_{\Omega} \mathbf{r}_{F}(\mathbf{v}) \cdot \boldsymbol{\tau}_{h} d \mathbf{x}=-\int_{F}\left\{\boldsymbol{\tau}_{h}\right\} \cdot \mathbf{v} d F \quad \forall \tau_{h} \in\left[\mathbb{P}_{d}^{k}\left(\mathcal{T}_{h}\right)\right]^{d}
$$

The global lifting operator $\mathbf{r}$ is then defined as

$$
\mathbf{r}(\mathbf{v}) \stackrel{\text { def }}{=} \sum_{F \in \mathcal{F}_{h}} \mathbf{r}_{F}(\mathbf{v}) .
$$

\subsection{DG discretization of the Navier-Stokes equations}

The Navier-Stokes equations for the $m$ variables in $d$ dimensions, Eqs. (1)-(2)-(3) or (7)-(8), can be written in compact form as

$$
\mathbf{P}(\mathbf{w}) \frac{\partial \mathbf{w}}{\partial t}+\nabla \cdot \mathbf{F}_{c}(\mathbf{w})+\nabla \cdot \mathbf{F}_{v}(\mathbf{w}, \nabla \mathbf{w})=\mathbf{0},
$$


where $\mathbf{w} \in \mathbb{R}^{m}$ is the unknown solution vector, $\mathbf{F}_{c}, \mathbf{F}_{v} \in \mathbb{R}^{m} \otimes \mathbb{R}^{d}$ are the convective and viscous flux functions, and $\mathbf{P}(\mathbf{w}) \in \mathbb{R}^{m} \otimes \mathbb{R}^{m}$ is a transformation matrix. Employing the conservative variables $\mathbf{w}_{c}=\left[\rho, \rho u_{i}, \rho E\right]^{T}$ for compressible flows and the primitive variables $\mathbf{w}_{p}=\left[p, u_{i}\right]^{T}$ for incompressible flows, $\mathbf{P}$ reduces to the identity matrix $(\mathbf{P}=\mathbf{I})$ in the former case and to the difference between the identity and a single-entry matrix $\left(\mathbf{P}=\mathbf{I}-\mathbf{J}^{11}\right)$ in the latter.

While the choice $\mathbf{w}_{p}=\left[p, u_{i}\right]^{T}$ is quite natural for incompressible flows, several reasons can lead to use a set of primitive variables also for compressible flows. For example, the set of primitive variables $\left(p, u_{i}, T\right)$ has long since been employed for preconditioning the governing equations of low Mach number compressible flows, $[45,46]$, and more recently has also been shown to be effective in the context of high-order DG approximations, [47]. Moreover, the primitive variables allow an easier and more efficient computation of the contributions to the Jacobian matrix related to the viscous terms discretization and to the implicit treatment of boundary conditions. The use of primitive variables for compressible flows offers also the interesting and useful possibility to ensure the positivity of all thermodynamic variables at the discrete level. For this purpose, it is in fact enough to work with polynomial approximations not directly for $p$ and $T$, but for their logarithms $\widetilde{p}=\log (p)$ and $\widetilde{T}=\log (T)$, [6]. In this way pressure and temperature values computed as $p=e^{\widetilde{p}}, T=e^{\widetilde{T}}$ are always positive. A numerical assessment of the effect of this change of variables on the solution accuracy is reported in Appendix A.

Using then, for compressible flows, the set of variables $\mathbf{w}=\left[\widetilde{p}, u_{i}, \widetilde{T}\right]^{T}$, the transformation matrix $\mathbf{P}(\mathbf{w})$ reads

$$
\mathbf{P}(\mathbf{w})=\left[\begin{array}{ccccc}
\rho_{\widetilde{p}} & 0 & 0 & 0 & \rho_{\widetilde{T}} \\
\rho_{\widetilde{p}} u_{1} & \rho & 0 & 0 & \rho_{\widetilde{T}} u_{1} \\
\rho_{\widetilde{p}} u_{2} & 0 & \rho & 0 & \rho_{\widetilde{T}} u_{2} \\
\rho_{\widetilde{p}} u_{3} & 0 & 0 & \rho & \rho_{\widetilde{T}} u_{3} \\
\rho_{\widetilde{p}} H+\rho h_{\widetilde{p}}-e^{\widetilde{p}} & \rho u_{1} & \rho u_{2} & \rho u_{3} & \rho_{\widetilde{T}} H+\rho h_{\widetilde{T}}
\end{array}\right],
$$

where

$$
\begin{gathered}
\rho=e^{(\widetilde{p}-\widetilde{T})}, \quad \hat{e}=\frac{e^{\widetilde{T}}}{\gamma-1}, \\
h_{\widetilde{p}}=\left.\frac{\partial h}{\partial \widetilde{p}}\right|_{\widetilde{T}}=\hat{e}_{\widetilde{p}}+\frac{e^{\widetilde{p}}}{\rho}-\frac{\rho_{\widetilde{p}}}{\rho^{2}} e^{\widetilde{p}}, \quad h_{\widetilde{T}}=\left.\frac{\partial h}{\partial \widetilde{T}}\right|_{\widetilde{p}}=\hat{e}_{\widetilde{T}}-\frac{\rho_{\widetilde{T}}}{\rho^{2}} e^{\widetilde{p}},
\end{gathered}
$$

and assuming an ideal gas

$$
\begin{gathered}
\rho_{\widetilde{p}}=\left.\frac{\partial \rho}{\partial \widetilde{p}}\right|_{\widetilde{T}}=\rho, \quad \rho_{\widetilde{T}}=\left.\frac{\partial \rho}{\partial \widetilde{T}}\right|_{\widetilde{p}}=-\rho, \\
\hat{e}_{\widetilde{p}}=\left.\frac{\partial \hat{e}}{\partial \widetilde{p}}\right|_{\widetilde{T}}=0, \quad \hat{e}_{\widetilde{T}}=\left.\frac{\partial \hat{e}}{\partial \widetilde{T}}\right|_{\widetilde{p}}=\hat{e}, \\
h_{\widetilde{p}}=0, \quad h_{\widetilde{T}}=\hat{e}_{\widetilde{T}}-\frac{e^{\widetilde{p}}}{\rho} .
\end{gathered}
$$

By multiplying Eq. (14) by an arbitrary smooth test function $\mathbf{v}=\left\{v_{1}, \ldots, v_{m}\right\}$, and integrating by parts, we obtain the weak formulation

$$
\int_{\Omega} \mathbf{v} \cdot\left(\mathbf{P}(\mathbf{w}) \frac{\partial \mathbf{w}}{\partial t}\right) d \mathbf{x}-\int_{\Omega} \nabla \mathbf{v}: \mathbf{F}(\mathbf{w}, \nabla \mathbf{w}) d \mathbf{x}+\int_{\partial \Omega} \mathbf{v} \otimes \mathbf{n}: \mathbf{F}(\mathbf{w}, \nabla \mathbf{w}) d \sigma=\mathbf{0},
$$


where $\mathbf{F}$ is the sum of the convective and viscous flux functions and $\mathbf{n}$ is the unit vector normal to the boundary.

To discretize Eq. (21) we replace the solution $\mathbf{w}$ and the test function $\mathbf{v}$ with a finite element approximation $\mathbf{w}_{h}$ and a discrete test function $\mathbf{v}_{h}$, respectively, where $\mathbf{w}_{h}$ and $\mathbf{v}_{h}$ belong to the space $\mathbf{V}_{h} \stackrel{\text { def }}{=}\left[\mathbb{P}_{d}^{k}\left(\mathcal{T}_{h}\right)\right]^{m}$. For each of the $m$ equations of system (21), and without loss of generality, we choose the set of test and shape functions in any element $K$ coincident with the set $\{\phi\}$ of $N_{d o f}^{K}$ orthogonal and hierachical basis functions in that element. With this choice each component $w_{h, j}, j=1, \ldots, m$, of $\mathbf{w}_{h} \in \mathbf{V}_{h}$ can be expressed, in terms of the elements of the global vector $\mathbf{W}$ of unknown degrees of freedom, as $w_{h, j}=\phi_{l} W_{j, l}, l=1, \ldots, N_{d o f}^{K}, \forall K \in \mathcal{T}_{h}$. Then, the DG discretization of the Navier-Stokes equations consists in seeking, for $j=1, \ldots, m$, the elements of $\mathbf{W}$ such that

$$
\begin{aligned}
\sum_{K \in \mathcal{T}_{h}} \int_{K} \phi_{i} P_{j, k}\left(\mathbf{w}_{h}\right) \phi_{l} \frac{d W_{k, l}}{d t} & d \mathbf{x}-\sum_{K \in \mathcal{T}_{h}} \int_{K} \frac{\partial \phi_{i}}{\partial x_{n}} F_{j, n}\left(\mathbf{w}_{h}, \nabla_{h} \mathbf{w}_{h}+\mathbf{r}\left(\llbracket \mathbf{w}_{h} \rrbracket\right)\right) d \mathbf{x} \\
& +\sum_{F \in \mathcal{F}_{h}} \int_{F} \llbracket \phi_{i} \rrbracket_{h} \widehat{F}_{j, n}\left(\mathbf{w}_{h}^{ \pm},\left(\nabla_{h} \mathbf{w}_{h}+\eta_{F} \mathbf{r}_{F}\left(\llbracket \mathbf{w}_{h} \rrbracket\right)\right)^{ \pm}\right) d \sigma=0
\end{aligned}
$$

for $i=1, \ldots, N_{d o f}^{K}$. In Eq. (22) repeated indices imply summation over the ranges $k=1, \ldots, m$, $l=1, \ldots, N_{d o f}^{K}, n=1, \ldots, d$.

The DG discretization of the viscous fluxes is based on the BR2 scheme, proposed in [36] and theoretically analyzed in [48] and [49]. According to this scheme, the viscous numerical flux is given by

$$
\widehat{\mathbf{F}}_{v}\left(\mathbf{w}_{h}^{ \pm},\left(\nabla_{h} \mathbf{w}_{h}+\eta_{F} \mathbf{r}_{F}\left(\llbracket \mathbf{w}_{h} \rrbracket\right)\right)^{ \pm}\right) \stackrel{\text { def }}{=}\left\{\mathbf{F}_{v}\left(\mathbf{w}_{h}, \nabla_{h} \mathbf{w}_{h}+\eta_{F} \mathbf{r}_{F}\left(\llbracket \mathbf{w}_{h} \rrbracket\right)\right)\right\}
$$

where the stability parameter $\eta_{F}$ is defined according to [49].

The convective numerical flux is computed from the solution of local Riemann problems in the normal direction at each integration point on elements faces. For compressible flows, we use either the exact Riemann solver of Gottlieb and Groth, [50], or, alternatively, the van Leer flux vector splitting method as modified by Hänel et al., [51]. For incompressible flows, we employ the approach proposed in [37], whereby the convective numerical flux is computed from the exact solution of local Riemann problems suitably modified by means of an artificial compressibility perturbation. According to the results of numerical experiments presented in [37] and [52], the value of the artificial compressibility parameter can be chosen in the range $[0.01,100]$, without affecting the numerical accuracy. For the incompressible flow computations presented in this work the value was set equal to 1 .

The DG discretization is very well suited to weakly enforce boundary conditions, [36, 53]. This can easily be achieved by properly defining at each integration point on boundaries the state $\mathbf{w}^{b}$ and its gradient $\nabla \mathbf{w}^{b}$, which are used, directly or together with the internal state and its gradient, to compute the numerical convective and viscous fluxes and the lifting operators for all $F \in \mathcal{F}_{h}^{b}$. At inflow, outflow, and farfield boundaries the state $\mathbf{w}^{b}$, which is defined using prescribed boundary data and the Riemann invariants related to the outgoing characteristics, enters in the Riemann solver and allows to compute, together with the internal state $\mathbf{w}_{h}^{-}$, the numerical convective flux. For these types of boundaries, the gradient $\nabla \mathbf{w}^{b}$ is defined as $\nabla \mathbf{w}^{b}=$ $\nabla \mathbf{w}_{h}^{-}+\eta_{F} \mathbf{r}_{F}\left(\llbracket \mathbf{w} \rrbracket^{b}\right)$, where $\mathbf{r}_{F}\left(\llbracket \mathbf{w} \rrbracket^{b}\right)$ is the lifting associated to the jump between $\mathbf{w}^{b}$ and $\mathbf{w}_{h}^{-}$. For solid wall boundaries, the state $\mathbf{w}^{b}$ and its gradient $\nabla \mathbf{w}^{b}$ are defined exactly at the wall. Hence, the state at the wall has zero velocity relative to the boundary and the pressure is determined from the 
analytical solution of the Riemann problem for a flow moving towards, or away from, the wall with the normal velocity component of the internal state. For compressible flows, the energy conservation equation requires a boundary condition either for the wall temperature, which is set in the state $\mathbf{w}^{b}$, or for the wall heat flux, which is proportional to the normal component of the temperature gradient $\nabla \mathbf{w}^{b}$ at the wall.

\subsection{Time integration}

Numerical integration of Eq. (22) by means of suitable Gauss quadrature rules leads to a system of nonlinear ODEs, or DAEs for incompressible flows, that can be written as

$$
\mathbf{M}_{\mathbf{P}}(\mathbf{W}) \frac{d \mathbf{W}}{d t}+\mathbf{R}(\mathbf{W})=\mathbf{0}
$$

where $\mathbf{R}(\mathbf{W})$ is the vector of residuals and $\mathbf{M}_{\mathbf{P}}(\mathbf{W})$ is a global block diagonal matrix arising from the discretization of the first term in Eq. (22). Thanks to the use of orthonormal basis functions defined in the physical space, the matrix $\mathbf{M}_{\mathbf{P}}$ reduces to the identity matrix for the compressible flow variables $\mathbf{w}_{c}=\left[\rho, \rho u_{i}, \rho E\right]^{T}$, and to a modified identity matrix, with zeros in the diagonal positions corresponding to the pressure degrees of freedom, for the incompressible flow variables $\mathbf{w}_{p}=\left[p, u_{i}\right]^{T}$. For different sets of variables, however, the transformation matrix $\mathbf{P}$ will couple the degrees of freedom of the variables $\mathbf{w}_{h}$ within each block of $\mathbf{M}_{\mathbf{P}}$, hence $\mathbf{M}_{\mathbf{P}}$ will no longer be diagonal. Below we describe the implementation we have adopted in order to account for the solution dependent block diagonal matrix $\mathbf{M}_{\mathbf{P}}(\mathbf{W})$ in linearly implicit Rosenbrock-type RungeKutta schemes.

The time integration of Eq. (24) by means of Rosenbrock schemes can be written as

$$
\begin{aligned}
\mathbf{W}^{n+1} & =\mathbf{W}^{n}+\sum_{j=1}^{s} b_{j} \mathbf{K}_{j}, \\
\left(\frac{\mathbf{I}}{\Delta t}+\gamma \mathbf{J}\right)^{n} \mathbf{K}_{i} & =-\widetilde{\mathbf{R}}\left(\mathbf{W}^{n}+\sum_{j=1}^{i-1} \alpha_{i j} \mathbf{K}_{j}\right)-\widetilde{\mathbf{J}}^{n} \sum_{j=1}^{i-1} \gamma_{i j} \mathbf{K}_{j}, \quad i=1, \ldots, s,
\end{aligned}
$$

where, omitting the dependence on $\mathbf{W}$ for notational convenience,

$$
\mathbf{J}=\frac{\partial \mathbf{R}}{\partial \mathbf{W}}, \quad \widetilde{\mathbf{R}}=\mathbf{M}_{\mathbf{P}}^{-1} \mathbf{R}, \quad \widetilde{\mathbf{J}}=\frac{\partial \widetilde{\mathbf{R}}}{\partial \mathbf{W}}=\mathbf{M}_{\mathbf{P}}^{-1}\left(\mathbf{J}-\frac{\partial \mathbf{M}_{\mathbf{P}}}{\partial \mathbf{W}} \widetilde{\mathbf{R}}\right),
$$

and $b_{i}, \alpha_{i j}, \gamma_{i j}$ are real coefficients. In this work the Jacobian matrix $\mathbf{J}$ of the DG space discretization is computed analytically and fully accounts for the dependence of the residuals on the unknown vector and its derivatives, including the implicit treatment of lifting operators and of boundary conditions. A direct implementation of Eq. (26) entails a matrix-vector product $\widetilde{\mathbf{J}}^{n} \sum_{j=1}^{i-1} \gamma_{i j} \mathbf{K}_{j}$ from the second stage on. In practice this can be avoided by noting that Eqs. (25) and (26) can be reduced to the following equivalent formulation, [25],

$$
\begin{gathered}
\mathbf{W}^{n+1}=\mathbf{W}^{n}+\sum_{j=1}^{s} m_{j} \mathbf{Y}_{j} \\
\left(\frac{\mathbf{I}}{\gamma \Delta t}+\widetilde{\mathbf{J}}\right)^{n} \mathbf{Y}_{i}=-\widetilde{\mathbf{R}}\left(\mathbf{W}^{n}+\sum_{j=1}^{i-1} a_{i j} \mathbf{Y}_{j}\right)+\sum_{j=1}^{i-1} \frac{c_{i j}}{\Delta t} \mathbf{Y}_{j}, \quad i=1, \ldots, s,
\end{gathered}
$$


where the quantities $\mathbf{K}_{i}$ and $\mathbf{Y}_{i}$ and the coefficients of the two formulations are related by the equations

$$
\begin{gathered}
\mathbf{K}_{i}=\frac{1}{\gamma} \mathbf{Y}_{i}-\sum_{j=1}^{i-1} c_{i j} \mathbf{Y}_{j}, \quad i=1, \ldots, s, \\
\left(m_{1}, \ldots, m_{s}\right)=\left(b_{1}, \ldots, b_{s}\right) \boldsymbol{\Gamma}^{-1}, \quad\left(a_{i j}\right)=\left(\alpha_{i j}\right) \boldsymbol{\Gamma}^{-1}, \quad\left(c_{i j}\right)=\gamma^{-1} \mathbf{I}_{s}-\boldsymbol{\Gamma}^{-1},
\end{gathered}
$$

and $\boldsymbol{\Gamma}^{-1} \stackrel{\text { def }}{=}\left(\gamma_{i j}\right)^{-1}$ denotes the inverse of the matrix formed with the coefficients $\left(\gamma_{i j}\right)$ of the schemes.

Recasting Eq. (29) so as to avoid the cumbersome product $\mathbf{M}_{\mathbf{P}}^{-1} \mathbf{J}$ in Eq. (27), we obtain the final form of the proposed implementation

$$
\begin{aligned}
\mathbf{W}^{n+1} & =\mathbf{W}^{n}+\sum_{j=1}^{s} m_{j} \mathbf{Y}_{j}, \\
\left(\frac{\mathbf{M}_{\mathbf{P}}}{\gamma \Delta t}+\mathbf{J}-\frac{\partial \mathbf{M}_{\mathbf{P}}}{\partial \mathbf{W}} \widetilde{\mathbf{R}}\right)^{n} \mathbf{Y}_{i} & =-\mathbf{M}_{\mathbf{P}}^{n}\left[\widetilde{\mathbf{R}}\left(\mathbf{W}^{n}+\sum_{j=1}^{i-1} a_{i j} \mathbf{Y}_{j}\right)-\sum_{j=1}^{i-1} \frac{c_{i j}}{\Delta t} \mathbf{Y}_{j}\right], \quad i=1, \ldots, s,
\end{aligned}
$$

which also allows to quantify the computational overhead due to the solution dependent block diagonal matrix $\mathbf{M}_{\mathbf{P}}$.

Eq. (31) is solved by means of the GMRES algorithm available in PETSc, [54], the library we employ also to manage parallel distributed arrays and the communication among processes. Preconditioning of the iterative solver is based on the block Jacobi method with one block per process, each of which is solved with ILU(0), or on the Additive Schwarz Method (ASM).

\subsubsection{Stability properties}

In this section we summarize the stability results for the Rosenbrock schemes considered in this paper and referred to as ROq-s, where $\mathrm{q}$ is the order of convergence and $\mathrm{s}$ is the number of stages. The schemes RO2-2 of Iannelli and Baker, [55], RO3-3 (ROS3P) of Lang and Verwer, [24], RO3-4 (RODAS3) of Hairer and Wanner, [25], RO4-6 (RODASP) of Steinebach, [26], and RO5-8 (RODAS5(4)-Rod5_1) of Di Marzo, [27], preserve their accuracy with DAEs. Instead, the schemes RO4-4 (ROS4) of Shampine, [28], and RO6-6 (ROW6A) of Kaps and Wanner, [29], are suited only for ODEs. For completeness, the sets of coefficients actually employed in Eqs. (30) and (31) are reported in Appendix B.

Figure 1 displays the stability area of the above schemes and allows to appreciate that all the schemes are A-stable. The stability areas have been obtained by means of the analytical procedure described by Hairer and Wanner in [25]. According to this procedure, the Rosenbrock schemes are applied to the following model equation

$$
\frac{d w}{d t}-\lambda w=0
$$

where the boundary of the stability domain $\mu$ is given by

$$
\mu=\lambda \Delta t,
$$

and the numerical solution at a given time step is set equal to

$$
\begin{gathered}
w^{n+t}=e^{\iota t \theta}, \\
8
\end{gathered}
$$




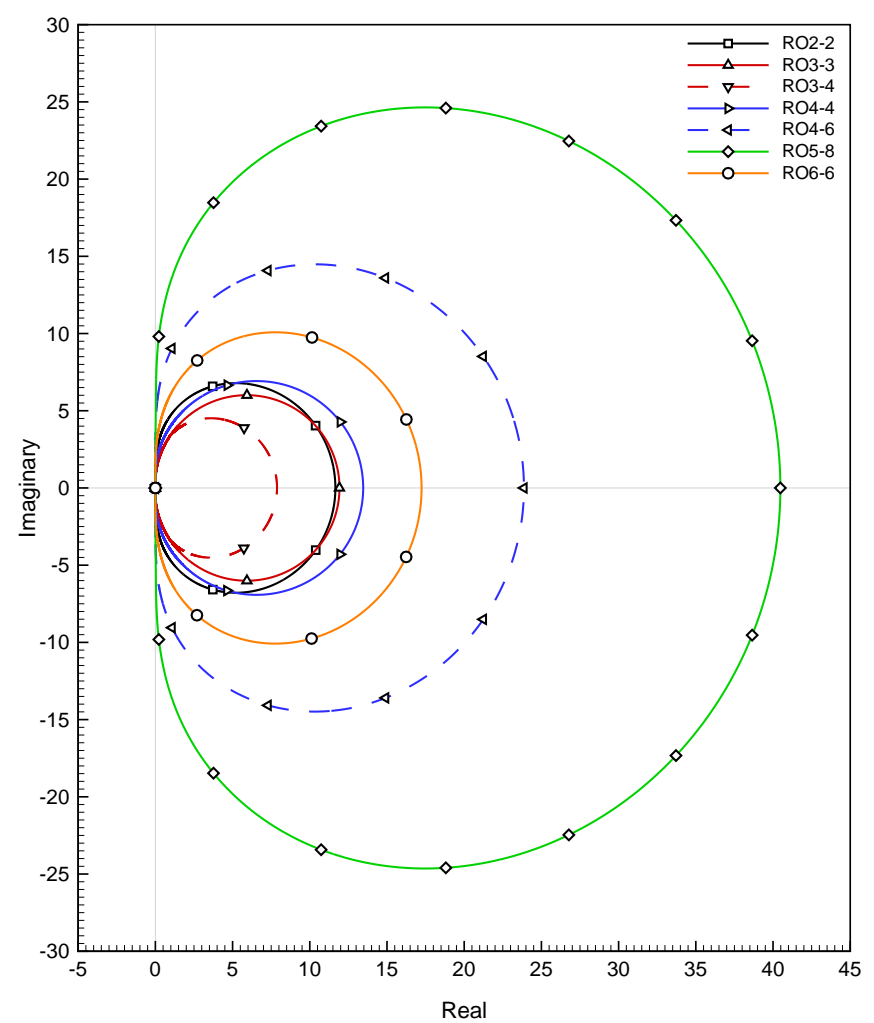

Figure 1: Stability area of the Rosenbrock schemes considered in this paper

where, to avoid ambiguity with the repeated indices notation, $\iota$ denotes the imaginary unit.

An $s$-stage Rosenbrock method applied to Eq. (32) reads

$$
\begin{gathered}
w^{n+1}=w^{n}+\sum_{i=1}^{s} m_{i} Y_{i} \\
\left(\frac{1}{\gamma \Delta t}-\lambda\right) Y_{i}=\lambda\left(w^{n}+\sum_{j=1}^{i-1} a_{i j} Y_{j}\right)+\sum_{j=1}^{i-1} \frac{c_{i j}}{\Delta t} Y_{j}, \quad i=1, \ldots, s .
\end{gathered}
$$

Using Eq. (33) we can write

$$
\begin{gathered}
Y_{i}=\frac{\sum_{j=1}^{i} d_{i j} \mu^{j}}{(1-\gamma \mu)^{i}} w^{n}, \quad i=1, \ldots, s, \\
\sum_{i=1}^{s} m_{i} Y_{i}=\frac{\sum_{k=1}^{s} \sum_{i=1}^{s} m_{i} g_{i k}^{(s)} \mu^{k}}{\sum_{k=0}^{s} f_{(s+1)(k+1)}(\gamma \mu)^{k}} w^{n},
\end{gathered}
$$

where the parameters $d_{i j}$ are functions of the Rosenbrock scheme coefficients only, $f_{i j}$ are the 
elements of the matrix $F \in \mathbb{R}^{(s+1) \times(s+1)}$

$$
f_{i j}= \begin{cases}1 & j=1 \\ 0 & i=1 \wedge j \neq 1 \\ f_{(i-1) j}-f_{(i-1)(j-1)} & \text { otherwise, }\end{cases}
$$

and $g_{i k}^{(s)}$ are the elements of the matrix $G^{(s)} \in \mathbb{R}^{s \times s}$ defined such that

$$
\sum_{k=1}^{s} g_{i k}^{(s)} \mu^{k}=\sum_{t=0}^{s-i} f_{(s-i+1)(t+1)}(\gamma \mu)^{t} \sum_{j=1}^{i} d_{i j} \mu^{j}, \quad i=1, \ldots, s .
$$

By inserting Eqs. (38) and (34) into Eq. (35) we finally obtain

$$
\sum_{k=0}^{s} A_{k}(\theta) \mu^{k}=0
$$

where

$$
A_{k}(\theta)= \begin{cases}1-e^{\iota \theta} & k=0 \\ \sum_{i=1}^{s} m_{i} g_{i k}^{(s)}+\left(1-e^{\iota \theta}\right) f_{(s+1)(k+1)} \gamma^{k} & \text { otherwise. }\end{cases}
$$

For $\theta \in[0 ; 2 \pi]$ the roots of Eq. $(41), \mu_{j}(\theta), j=1, \ldots, s$, describe the stability area of a Rosenbrock scheme with $s$ stages. Expressions for the $d_{i j}$ parameters and the elements of the $G^{(s)}$ matrices up to $s=4$ are reported in Appendix C.

\section{Numerical Results}

The performance and the order of convergence of the Rosenbrock schemes introduced in Sec. 3.3 have been numerically assessed on two simple test cases of compressible and incompressible flow. One of the best performing schemes has then been applied to the high-order DG Implicit Large-Eddy Simulation (ILES) of an external aerodynamic flow problem.

\subsection{Convection of an isentropic vortex}

An inviscid isentropic vortex transported by a uniform flow, [56, 20, 6], is here considered. The vortex is defined by velocity and temperature perturbations of a uniform flow, with pressure, temperature and density equal to 1 and with velocity components equal to $\sqrt{\gamma}$, where $\gamma=1.4$ is the ratio of specific heats. The perturbations are given by

$$
\delta u_{1}=-\frac{\alpha}{2 \pi}\left(y-y_{0}\right) e^{\phi\left(1-r^{2}\right)}, \quad \delta u_{2}=\frac{\alpha}{2 \pi}\left(x-x_{0}\right) e^{\phi\left(1-r^{2}\right)}, \quad \delta T=-\frac{\alpha^{2}(\gamma-1)}{16 \phi \gamma \pi^{2}} e^{2 \phi\left(1-r^{2}\right)},
$$

where $\phi=\frac{1}{2}$ and $\alpha=5$ are parameters defining the vortex strength, $r$ is the distance of a point $(x, y)$ from the vortex center $\left(x_{0}, y_{0}\right)$, placed at $(5,5)$ in a periodic domain $[0,10] \times[0,10]$ at time $t_{0}=0$. The initial pressure field is shown in Figure 2(a). Notice that, due to the periodic boundary conditions, the problem actually solved is that of a regular grid of vortices moving on and parallel to the domain diagonal. As observed by Nigro et al., [20], it is important to take into account the contributions of nearby vortices on the initial and reference solutions, otherwise a reduced rate of convergence will be observed for sufficiently accurate solutions. 


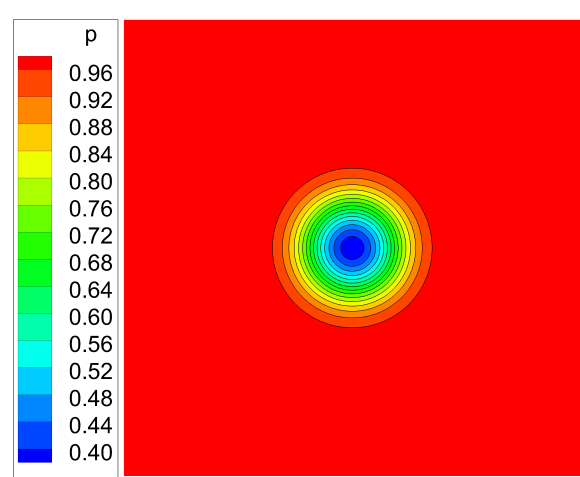

(a) Isentropic vortex - pressure

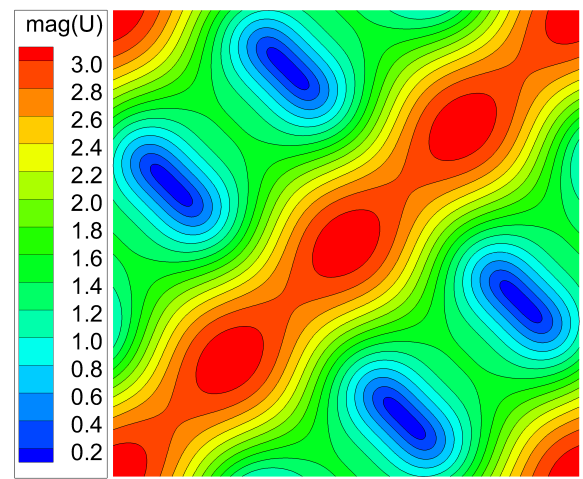

(b) Travelling waves - velocity magnitude

Figure 2: Isentropic vortex and travelling waves - initial flow field, DG $\mathbb{P}^{6}$ approximation (70000 DOFs)

To verify the order of convergence of the Rosenbrock schemes, the solutions have been computed using a very accurate $\mathbb{P}^{6} \mathrm{DG}$ space discretization on a uniform $50 \times 50$ quad mesh, resulting in 70000 degrees of freedom per equation (DOFs). The GMRES solver parameters, i.e., number of vectors in the Krylov subspace $n_{r s t}$, maximum number of iterations $n_{\max }$, and tolerance on the relative residual norm $\operatorname{tol}_{r}$, have been set to $n_{r s t}=120, n_{\max }=240$, and $t_{o} l_{r}=1 \mathrm{e}-14$. The results reported in Table 1 and shown in Figure 3 demonstrate that all the Rosenbrock schemes verify the formal order of convergence for all the variables.

In Table 1 and Figure 4 the computational time of the Rosenbrock schemes is given in terms of Work Units (WU) defined by WU $=t_{\text {wall }} N_{\text {cores }} / t_{T B}$, where $t_{\text {wall }}$ is the execution time of a simulation on $N_{\text {cores }}$ cores and $t_{T B}$ is the reference TauBenchmark time of the hardware ${ }^{1}$. These results show that, for a given order of convergence and among the schemes here considered, those with more stages are more accurate and efficient. The large number of stages even makes the RO5-8 scheme competitive with the formally more accurate RO6-6 scheme.

In order to assess the sensitivity of the computational cost of the schemes to tol $_{r}$, this tolerance has been increased, for each scheme, up to the value for which the convergence rate differs by less than $1 \%$ from the convergence rate with $\operatorname{tol}_{r}=1 \mathrm{e}-14$. The results reported in Table 1 show that increasing tol $_{r}$ entails a significant reduction of the computational cost for all the schemes, without affecting the convergence rate. Overall, the results of this test case show that higherorder Rosenbrock schemes are much more efficient than lower-order ones when coupled to a high-order DG space discretization.

\subsection{Incompressible travelling waves}

For incompressible flows, the accuracy and performance of the time integration schemes has been assessed on the following travelling-wave solution to the Navier-Stokes equations on the

\footnotetext{
${ }^{1}$-n 250000 -s 10 define the reference TauBench workload for the hardware benchmark
} 
Table 1: Isentropic vortex, ROq-s-DG $\mathbb{P}^{6}$ solutions $-L^{2}$ norm of the error with respect to the exact solution for pressure and temperature, convergence rate, Work Units (WU), maximum $t_{0} l_{r}$ ensuring a convergence rate close to (within $1 \%$ ) the convergence rate with $\operatorname{tol}_{r}=1 \mathrm{e}-14\left(\operatorname{tol}_{1} \%\right)$, computational cost percentage reduction with respect to $t_{0} l_{r}=1 \mathrm{e}-14$ $(\Delta \% \mathrm{WU})$.

\begin{tabular}{|c|c|c|c|c|c|c|c|c|}
\hline & $\Delta t$ & $\left\|e r r_{p}\right\|_{2}$ & $\operatorname{order}_{p}$ & $\left\|e r r_{T}\right\|_{2}$ & $\operatorname{order}_{T}$ & WU & $\frac{50}{9}$ & $\Delta \% \mathrm{WU}$ \\
\hline \multirow{5}{*}{ 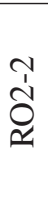 } & $T / 125$ & $1.427 \mathrm{e}-3$ & - & $1.834 \mathrm{e}-3$ & - & 1087.7 & \multirow{5}{*}{$\stackrel{m}{\jmath}$} & -30.1 \\
\hline & $T / 160$ & $8.694 \mathrm{e}-4$ & 2.008 & $1.116 \mathrm{e}-3$ & 2.012 & 1350.7 & & -28.5 \\
\hline & $T / 200$ & $5.557 \mathrm{e}-4$ & 2.006 & $7.129 \mathrm{e}-4$ & 2.008 & 1634.1 & & -24.4 \\
\hline & $T / 250$ & $3.553 \mathrm{e}-4$ & 2.004 & $4.557 \mathrm{e}-4$ & 2.006 & 2010.5 & & -25.0 \\
\hline & $T / 320$ & $2.167 \mathrm{e}-4$ & 2.003 & $2.779 \mathrm{e}-4$ & 2.004 & 2512.5 & & -23.3 \\
\hline \multirow{5}{*}{ 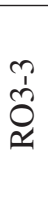 } & $T / 125$ & $7.756 e-4$ & - & $3.985 \mathrm{e}-4$ & - & 1588.4 & \multirow{5}{*}{$\begin{array}{l}+ \\
\stackrel{1}{0} \\
\end{array}$} & -40.3 \\
\hline & $T / 160$ & $3.773 \mathrm{e}-4$ & 2.919 & $2.013 \mathrm{e}-4$ & 2.767 & 1969.9 & & -38.4 \\
\hline & $T / 200$ & $1.954 \mathrm{e}-4$ & 2.949 & $1.068 \mathrm{e}-4$ & 2.838 & 2304.3 & & -26.5 \\
\hline & $T / 250$ & $1.008 \mathrm{e}-4$ & 2.966 & $5.606 \mathrm{e}-5$ & 2.890 & 2894.2 & & -30.1 \\
\hline & $T / 320$ & $4.834 \mathrm{e}-5$ & 2.978 & $2.720 \mathrm{e}-5$ & 2.929 & 3393.4 & & -32.6 \\
\hline \multirow{5}{*}{ ֻُ } & $T / 125$ & $1.882 \mathrm{e}-4$ & - & $2.641 \mathrm{e}-4$ & - & 1712.8 & \multirow{5}{*}{$\begin{array}{l}+ \\
\vdots \\
0\end{array}$} & -40.8 \\
\hline & $T / 160$ & $9.022 \mathrm{e}-5$ & 2.978 & $1.270 \mathrm{e}-4$ & 2.966 & 2108.6 & & -39.0 \\
\hline & $T / 200$ & $4.633 e-5$ & 2.987 & $6.533 e-5$ & 2.978 & 2478.4 & & -35.8 \\
\hline & $T / 250$ & $2.377 \mathrm{e}-5$ & 2.991 & $3.356 \mathrm{e}-5$ & 2.986 & 2983.2 & & -34.4 \\
\hline & $T / 320$ & $1.135 \mathrm{e}-5$ & 2.994 & $1.604 \mathrm{e}-5$ & 2.990 & 3648.0 & & -31.7 \\
\hline \multirow{5}{*}{ 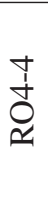 } & $T / 125$ & $2.555 \mathrm{e}-5$ & - & $4.238 \mathrm{e}-5$ & - & 1712.9 & \multirow{5}{*}{$\begin{array}{l}n \\
\varrho \\
ٍ\end{array}$} & -34.7 \\
\hline & $T / 160$ & $9.737 \mathrm{e}-6$ & 3.908 & $1.607 \mathrm{e}-6$ & 3.929 & 2100.5 & & -35.3 \\
\hline & $T / 200$ & $4.054 \mathrm{e}-6$ & 3.927 & $6.663 e-6$ & 3.945 & 2484.4 & & -32.5 \\
\hline & $T / 250$ & $1.681 \mathrm{e}-6$ & 3.945 & $2.754 \mathrm{e}-6$ & 3.959 & 3011.0 & & -31.4 \\
\hline & $T / 320$ & $6.322 \mathrm{e}-7$ & 3.961 & $1.034 \mathrm{e}-6$ & 3.971 & 3665.0 & & -32.0 \\
\hline \multirow{5}{*}{\begin{tabular}{l}
0 \\
$\stackrel{1}{1}$ \\
$\dot{0}$ \\
\multirow{1}{*}{}
\end{tabular}} & $T / 125$ & $1.602 \mathrm{e}-6$ & - & $8.047 e-7$ & - & 1916.7 & \multirow{5}{*}{$\begin{array}{l}0 \\
1 \\
0\end{array}$} & -32.7 \\
\hline & $T / 160$ & $6.002 \mathrm{e}-7$ & 3.978 & $3.009 \mathrm{e}-7$ & 3.985 & 2297.1 & & -31.2 \\
\hline & $T / 200$ & $2.467 \mathrm{e}-7$ & 3.984 & $1.235 \mathrm{e}-7$ & 3.990 & 2791.0 & & -30.0 \\
\hline & $T / 250$ & $1.013 \mathrm{e}-7$ & 3.989 & $5.068 \mathrm{e}-8$ & 3.993 & 3269.8 & & -27.5 \\
\hline & $T / 320$ & $3.781 \mathrm{e}-8$ & 3.992 & $1.890 \mathrm{e}-8$ & 3.995 & 4124.6 & & -28.0 \\
\hline \multirow{5}{*}{ 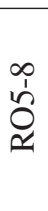 } & $T / 125$ & $3.517 \mathrm{e}-8$ & - & $6.627 \mathrm{e}-8$ & - & 2216.3 & \multirow{5}{*}{$\begin{array}{l}\infty \\
1 \\
0\end{array}$} & -24.8 \\
\hline & $T / 160$ & $1.005 \mathrm{e}-8$ & 5.075 & $2.044 \mathrm{e}-8$ & 4.793 & 2683.9 & & -23.8 \\
\hline & $T / 200$ & $3.244 \mathrm{e}-9$ & 5.067 & $6.894 \mathrm{e}-9$ & 4.870 & 3881.2 & & -21.5 \\
\hline & $T / 250$ & $1.051 \mathrm{e}-9$ & 5.052 & $2.304 \mathrm{e}-9$ & 4.912 & 4750.7 & & -21.5 \\
\hline & $T / 320$ & $3.323 e-10$ & 4.663 & $6.888 \mathrm{e}-10$ & 4.890 & 4750.7 & & -19.1 \\
\hline \multirow{5}{*}{$\begin{array}{l}0 \\
1 \\
0 \\
0\end{array}$} & $T / 125$ & $1.891 \mathrm{e}-7$ & - & $1.271 \mathrm{e}-7$ & - & 2103.6 & \multirow{5}{*}{$\begin{array}{l}\infty \\
1 \\
0\end{array}$} & -26.1 \\
\hline & $T / 160$ & $4.506 \mathrm{e}-8$ & 5.810 & $2.954 \mathrm{e}-8$ & 5.912 & 2474.4 & & -25.1 \\
\hline & $T / 200$ & $1.189 \mathrm{e}-8$ & 5.972 & $7.549 \mathrm{e}-9$ & 6.114 & 3054.6 & & -22.9 \\
\hline & $T / 250$ & $3.094 \mathrm{e}-9$ & 6.031 & $1.891 \mathrm{e}-9$ & 6.203 & 3527.8 & & -23.2 \\
\hline & $T / 320$ & $7.212 \mathrm{e}-10$ & 5.899 & $4.196 \mathrm{e}-10$ & 6.099 & 4343.6 & & -20.4 \\
\hline
\end{tabular}


Table 1: Isentropic vortex, ROq-s-DG $\mathbb{P}^{6}$ solutions - $L^{2}$ norm of the error with respect to the exact solution for the velocity components, convergence rate, Work Units (WU), maximum tol $_{r}$ ensuring a convergence rate close to (within $1 \%$ ) the convergence rate with $t_{o} l_{r}=1 \mathrm{e}-14\left(\right.$ tol $\left._{1 \%}\right)$, computational cost percentage reduction with respect to tol $_{r}=1 \mathrm{e}-14$ $(\Delta \% \mathrm{WU})$.

\begin{tabular}{|c|c|c|c|c|c|c|c|c|}
\hline & $\Delta t$ & $\left\|e r r_{u_{1}}\right\|_{2}$ & $\operatorname{order}_{u_{1}}$ & $\left\|e r r_{u_{2}}\right\|_{2}$ & $\operatorname{order}_{u_{2}}$ & WU & $\frac{50}{9}$ & $\Delta \% \mathrm{WU}$ \\
\hline \multirow{5}{*}{ 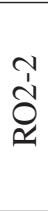 } & $T / 125$ & $3.324 \mathrm{e}-3$ & - & $1.834 \mathrm{e}-3$ & - & 1087.7 & \multirow{5}{*}{$\stackrel{m}{\jmath}$} & -30.1 \\
\hline & $T / 160$ & $2.026 \mathrm{e}-3$ & 2.007 & $1.116 \mathrm{e}-3$ & 2.012 & 1350.7 & & -28.5 \\
\hline & $T / 200$ & $1.295 \mathrm{e}-3$ & 2.005 & $7.129 \mathrm{e}-4$ & 2.008 & 1634.1 & & -24.4 \\
\hline & $T / 250$ & $8.282 \mathrm{e}-4$ & 2.003 & $4.557 \mathrm{e}-4$ & 2.006 & 2010.5 & & -25.0 \\
\hline & $T / 320$ & $5.052 \mathrm{e}-4$ & 2.002 & $2.779 \mathrm{e}-4$ & 2.004 & 2512.5 & & -23.3 \\
\hline \multirow{5}{*}{ 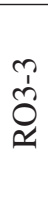 } & $T / 125$ & $1.376 \mathrm{e}-3$ & - & $1.098 \mathrm{e}-3$ & - & 1588.4 & \multirow{5}{*}{$\begin{array}{l}+ \\
\stackrel{1}{0} \\
\end{array}$} & -40.3 \\
\hline & $T / 160$ & $6.678 \mathrm{e}-4$ & 2.928 & $5.376 \mathrm{e}-4$ & 2.767 & 1969.9 & & -38.4 \\
\hline & $T / 200$ & $3.456 \mathrm{e}-4$ & 2.952 & $2.794 \mathrm{e}-4$ & 2.838 & 2304.3 & & -26.5 \\
\hline & $T / 250$ & $1.782 \mathrm{e}-4$ & 2.967 & $1.444 \mathrm{e}-4$ & 2.890 & 2894.2 & & -30.1 \\
\hline & $T / 320$ & $8.542 \mathrm{e}-5$ & 2.979 & $6.929 \mathrm{e}-5$ & 2.929 & 3393.4 & & -32.6 \\
\hline \multirow{5}{*}{ ֶֻ } & $T / 125$ & $3.363 e-4$ & - & $2.641 \mathrm{e}-4$ & - & 1712.8 & \multirow{5}{*}{$\begin{array}{l}+ \\
\text { I } \\
0\end{array}$} & -40.8 \\
\hline & $T / 160$ & $1.612 \mathrm{e}-4$ & 2.979 & $1.270 \mathrm{e}-4$ & 2.966 & 2108.6 & & -39.0 \\
\hline & $T / 200$ & $8.275 \mathrm{e}-5$ & 2.987 & $6.533 e-5$ & 2.978 & 2478.4 & & -35.8 \\
\hline & $T / 250$ & $4.244 \mathrm{e}-5$ & 2.992 & $3.356 \mathrm{e}-5$ & 2.986 & 2983.2 & & -34.4 \\
\hline & $T / 320$ & $2.027 \mathrm{e}-5$ & 2.995 & $1.604 \mathrm{e}-5$ & 2.990 & 3648.0 & & -31.7 \\
\hline \multirow{5}{*}{ 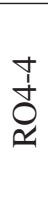 } & $T / 125$ & $5.223 e-5$ & - & $4.238 \mathrm{e}-5$ & - & 1712.9 & \multirow{5}{*}{ 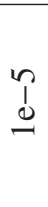 } & -34.7 \\
\hline & $T / 160$ & $1.972 \mathrm{e}-5$ & 3.947 & $1.607 \mathrm{e}-5$ & 3.929 & 2100.5 & & -35.3 \\
\hline & $T / 200$ & $8.150 \mathrm{e}-6$ & 3.959 & $6.663 \mathrm{e}-6$ & 3.945 & 2484.4 & & -32.5 \\
\hline & $T / 250$ & $3.362 \mathrm{e}-6$ & 3.968 & $2.754 \mathrm{e}-6$ & 3.959 & 3011.0 & & -31.4 \\
\hline & $T / 320$ & $1.260 \mathrm{e}-6$ & 3.976 & $1.034 \mathrm{e}-6$ & 3.971 & 3665.0 & & -32.0 \\
\hline \multirow{5}{*}{ 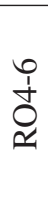 } & $T / 125$ & $3.309 \mathrm{e}-6$ & - & $2.795 \mathrm{e}-6$ & - & 1916.7 & \multirow{5}{*}{$\begin{array}{l}0 \\
1 \\
0\end{array}$} & -32.7 \\
\hline & $T / 160$ & $1.238 \mathrm{e}-6$ & 3.984 & $1.046 \mathrm{e}-7$ & 3.981 & 2297.1 & & -31.2 \\
\hline & $T / 200$ & $5.082 \mathrm{e}-7$ & 3.989 & $4.298 \mathrm{e}-7$ & 3.986 & 2791.0 & & -30.0 \\
\hline & $T / 250$ & $2.085 \mathrm{e}-7$ & 3.992 & $1.765 \mathrm{e}-7$ & 3.990 & 3269.8 & & -27.5 \\
\hline & $T / 320$ & $7.779 e-8$ & 3.994 & $6.586 \mathrm{e}-8$ & 3.992 & 4124.6 & & -28.0 \\
\hline \multirow{5}{*}{$\begin{array}{l}\infty \\
1 \\
\\
0 \\
\end{array}$} & $T / 125$ & $6.815 \mathrm{e}-8$ & - & $7.101 \mathrm{e}-8$ & - & 2216.3 & \multirow{5}{*}{$\begin{array}{l}\infty \\
1 \\
0\end{array}$} & -24.8 \\
\hline & $T / 160$ & $2.018 \mathrm{e}-8$ & 4.930 & $2.080 \mathrm{e}-8$ & 4.974 & 2683.1 & & -23.8 \\
\hline & $T / 200$ & $6.743 \mathrm{e}-9$ & 4.913 & $6.863 \mathrm{e}-9$ & 4.968 & 3881.2 & & -21.5 \\
\hline & $T / 250$ & $2.328 \mathrm{e}-9$ & 4.766 & $2.321 \mathrm{e}-9$ & 4.859 & 4750.7 & & -21.5 \\
\hline & $T / 320$ & $9.248 \mathrm{e}-10$ & 3.740 & $8.959 \mathrm{e}-10$ & 3.856 & 4750.7 & & -19.1 \\
\hline \multirow{5}{*}{ 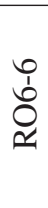 } & $T / 125$ & $4.129 \mathrm{e}-7$ & - & $3.611 \mathrm{e}-7$ & - & 2103.6 & \multirow{5}{*}{$\begin{array}{l}\infty \\
1 \\
0\end{array}$} & -26.1 \\
\hline & $T / 160$ & $9.218 \mathrm{e}-8$ & 6.074 & $8.091 \mathrm{e}-8$ & 6.060 & 2474.4 & & -25.1 \\
\hline & $T / 200$ & $2.276 \mathrm{e}-8$ & 6.269 & $2.005 \mathrm{e}-8$ & 6.252 & 3054.6 & & -22.9 \\
\hline & $T / 250$ & $5.533 \mathrm{e}-9$ & 6.337 & $4.906 \mathrm{e}-9$ & 6.306 & 3527.8 & & -23.2 \\
\hline & $T / 320$ & $1.300 \mathrm{e}-9$ & 5.868 & $1.191 \mathrm{e}-9$ & 5.734 & 4343.6 & & -20.4 \\
\hline
\end{tabular}



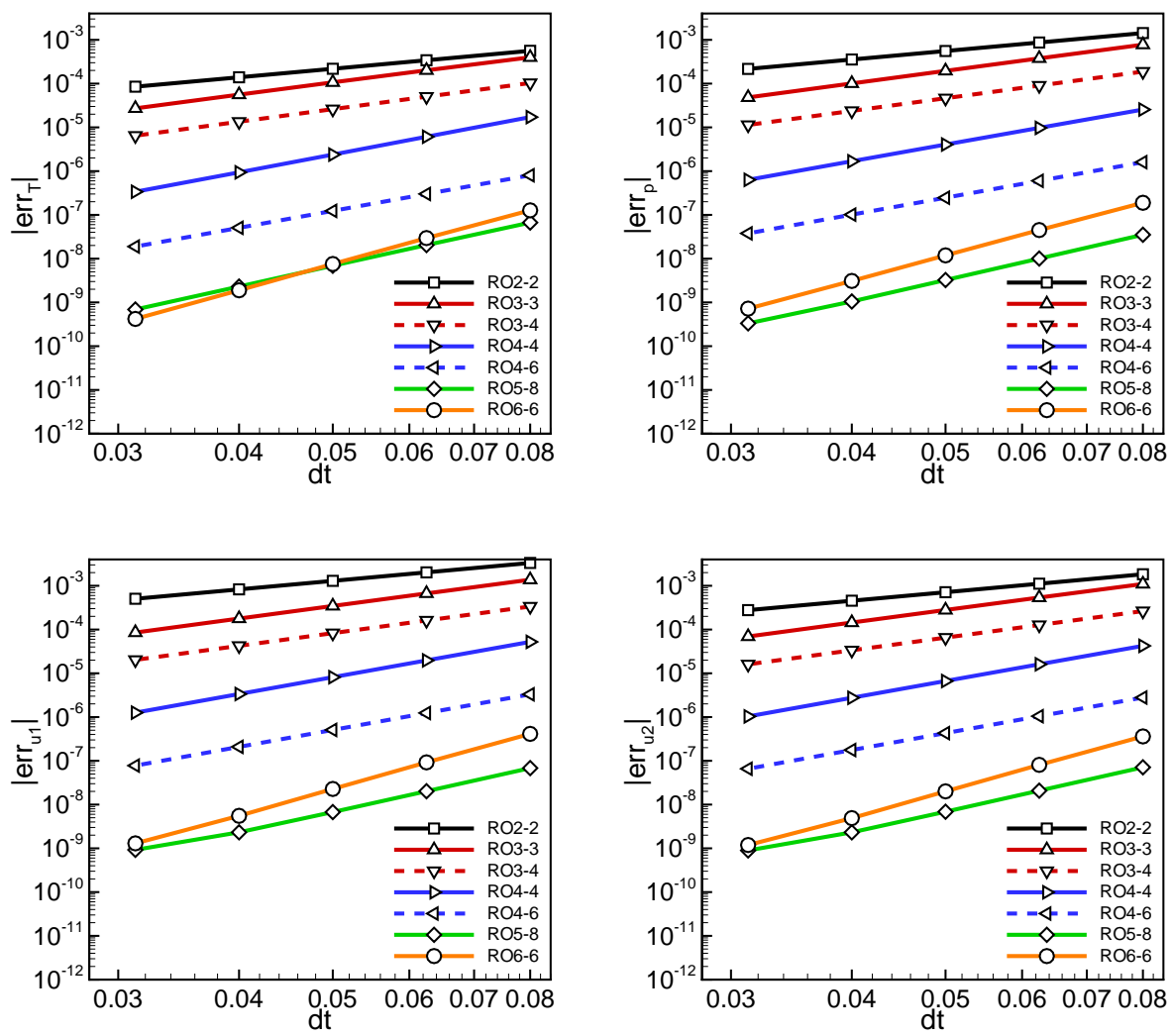

Figure 3: Isentropic vortex, ROq-s-DG $\mathbb{P}^{6}$ solutions $-L^{2}$ norm of the flow variables error vs. time step

doubly-periodic unit square $[0.25,1.25] \times[0.5,1.5]$

$$
\begin{aligned}
& u_{1}(x, y, t)=1+2 \cos (2 \pi(x-t)) \sin (2 \pi(y-t)) e^{-8 \pi^{2} v t}, \\
& u_{2}(x, y, t)=1-2 \sin (2 \pi(x-t)) \cos (2 \pi(y-t)) e^{-8 \pi^{2} v t}, \\
& p(x, y, t)=-(\cos (4 \pi(x-t))+\cos (4 \pi(y-t))) e^{-16 \pi^{2} v t,}
\end{aligned}
$$

with $v=1 \mathrm{e}-2$. The initial velocity field is shown in Figure 2(b). The solution has been computed on a uniform $50 \times 50$ quad mesh, using a $\mathbb{P}^{6}$ DG space approximation (70000 DOFs), enough to keep the space discretization error well below the time integration error and to reliably assess the order of convergence of the time integration schemes, [53]. In the incompressible case, the convergence of the iterative solver was more difficult than in the compressible case and the GMRES parameters, $n_{r s t}$ and $n_{\max }$, had to be set to quite large values, namely $n_{r s t}=480$ and $n_{\max }=960$, in order to satisfy the tolerance $t o l_{r}=1 \mathrm{e}-14$. The important issue of improving the efficiency of the GMRES solver in the incompressible case was beyond the scope of this paper and will be addressed in a future work.

The results reported in Table 2 and shown in Figure 5 prove that the Rosenbrock schemes designed for DAEs verify the formal order of convergence for all the variables, while, as expected, 

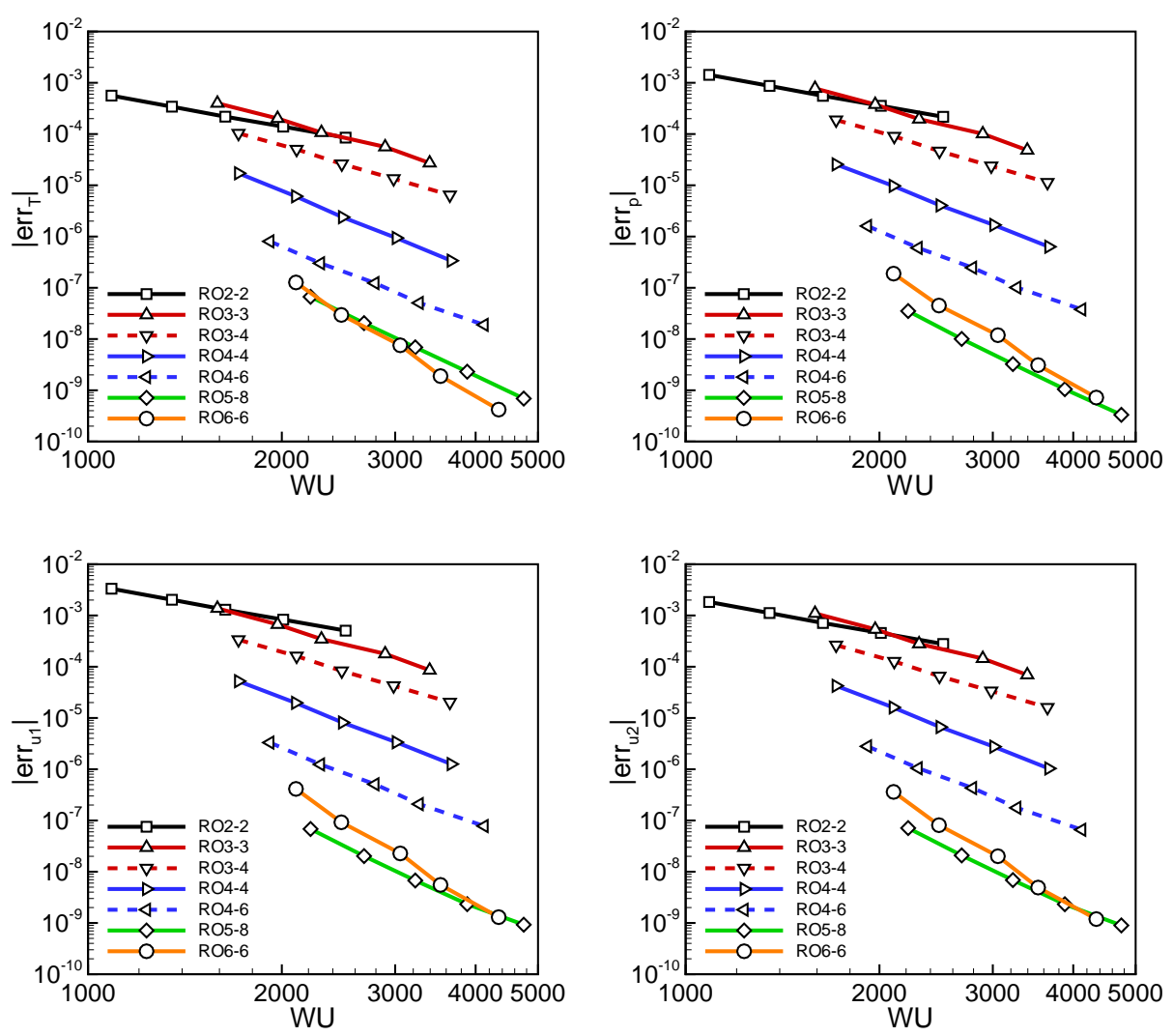

Figure 4: Isentropic vortex ROq-S-DG $\mathbb{P}^{6}$ solutions - $L^{2}$ norm of the error with respect to the exact solution vs. Work Units(WU)

the RO4-4 and RO6-6 schemes, designed for ODEs, exhibit order reduction for the $L^{2}$ error norm on pressure.

The values $\Delta \% \mathrm{WU}$ in Table 2 highlight the substantial reduction of computational cost related to the increase of the acceptable values of relative tolerance tol $_{1 \%}$ for the Rosenbrock schemes up to order 4. Notice that, compared to the compressible test case, the values of tol $_{1 \%}$ are smaller, while the reduction of the computational cost is much larger. This can be explained by considering that, in the incompressible case, the computational cost of the GMRES iterations is a comparatively large part of the overall computational cost in one time step, due to the large number of iterations required to satisfy the reference tolerance $t o l_{r}=1 \mathrm{e}-14$. On the other hand, since tol $_{1 \%}$ for the scheme RO5-8 is quite close to $\operatorname{tol}_{r}=1 \mathrm{e}-14, \Delta \% \mathrm{WU}$ is rather small.

As for the compressible isentropic vortex test case, the numerical experiments confirm that higher-order time integration schemes are preferable in terms of efficiency and accuracy.

\subsection{Transitional flow around the SD7003 airfoil}

The reliability of the proposed implicit and globally high-order approach to the simulation of unsteady flows is here demonstrated on the ILES of the transitional flow around the SD7003 
Table 2: Travelling waves, ROq-s-DG $\mathbb{P}^{6}$ solutions $-L^{2}$ norm of the error with respect to the exact solution for pressure and $\mathrm{x}-\mathrm{y}$-component of velocity, Work Units (WU), maximum tol $_{r}$ ensuring a convergence rate close to (within $1 \%$ ) the convergence rate with $\operatorname{tol}_{r}=1 \mathrm{e}-14\left(\right.$ tol $\left._{1} \%\right)$, computational cost percentage reduction with respect to $t l_{r}=1 \mathrm{e}-14$ $(\Delta \% \mathrm{WU})$.

\begin{tabular}{|c|c|c|c|c|c|c|c|c|}
\hline & $\Delta t$ & $\left\|\operatorname{err}_{p}\right\|_{2}$ & $\operatorname{order}_{p}$ & $\left\|\operatorname{err}_{u_{1,2}}\right\|_{2}$ & $\operatorname{order}_{u_{1,2}}$ & WU & $\frac{50}{9}$ & $\Delta \% \mathrm{WU}$ \\
\hline \multirow{5}{*}{ 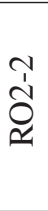 } & $T / 50$ & $6.119 \mathrm{e}-3$ & - & $1.036 \mathrm{e}-2$ & - & 4130.3 & \multirow{5}{*}{$\begin{array}{l}0 \\
1 \\
0\end{array}$} & -88.2 \\
\hline & $T / 80$ & $2.392 \mathrm{e}-3$ & 1.999 & $4.050 \mathrm{e}-3$ & 1.998 & 6722.9 & & -88.1 \\
\hline & $T / 100$ & $1.531 \mathrm{e}-3$ & 2.000 & $2.592 \mathrm{e}-3$ & 1.999 & 8567.2 & & -88.1 \\
\hline & $T / 160$ & $5.981 \mathrm{e}-4$ & 2.000 & $1.013 \mathrm{e}-3$ & 2.000 & 14720.7 & & -88.2 \\
\hline & $T / 200$ & $3.827 \mathrm{e}-4$ & 2.000 & $6.481 \mathrm{e}-4$ & 2.000 & 18489.9 & & -87.9 \\
\hline \multirow{5}{*}{ 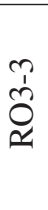 } & $T / 50$ & $3.460 \mathrm{e}-3$ & - & $5.439 \mathrm{e}-3$ & - & 6753.5 & \multirow{5}{*}{$\begin{array}{l}0 \\
1 \\
0\end{array}$} & -87.5 \\
\hline & $T / 80$ & $8.750 \mathrm{e}-4$ & 2.925 & $1.376 \mathrm{e}-3$ & 2.925 & 9983.6 & & -88.5 \\
\hline & $T / 100$ & $4.519 \mathrm{e}-4$ & 2.961 & $7.104 \mathrm{e}-4$ & 2.961 & 12763.5 & & -89.0 \\
\hline & $T / 160$ & $1.114 \mathrm{e}-4$ & 2.979 & $1.752 \mathrm{e}-4$ & 2.979 & 23244.2 & & -89.1 \\
\hline & $T / 200$ & $5.721 \mathrm{e}-5$ & 2.988 & $8.993 e-5$ & 2.988 & 25591.6 & & -90.2 \\
\hline \multirow{5}{*}{ 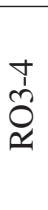 } & $T / 50$ & $8.513 e-4$ & - & $1.326 \mathrm{e}-3$ & - & 9931.6 & \multirow{5}{*}{$\begin{array}{l}0 \\
1 \\
0 \\
0\end{array}$} & -90.7 \\
\hline & $T / 80$ & $2.094 \mathrm{e}-4$ & 2.984 & $3.262 \mathrm{e}-4$ & 2.984 & 17548.1 & & -92.0 \\
\hline & $T / 100$ & $1.074 \mathrm{e}-4$ & 2.992 & $1.673 \mathrm{e}-4$ & 2.992 & 22178.1 & & -92.1 \\
\hline & $T / 160$ & $2.629 \mathrm{e}-5$ & 2.995 & $4.094 \mathrm{e}-5$ & 2.995 & 40257.5 & & -92.3 \\
\hline & $T / 200$ & $1.347 \mathrm{e}-5$ & 2.997 & $2.097 \mathrm{e}-5$ & 2.997 & 53782.4 & & -92.5 \\
\hline \multirow{5}{*}{ 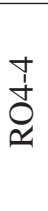 } & $T / 50$ & $5.059 \mathrm{e}-4$ & - & $1.932 \mathrm{e}-4$ & - & 8096.0 & \multirow{5}{*}{$\stackrel{1}{1}$} & -86.2 \\
\hline & $T / 80$ & $1.775 \mathrm{e}-4$ & 2.229 & $3.024 \mathrm{e}-5$ & 3.945 & 13623.5 & & -87.4 \\
\hline & $T / 100$ & $1.101 \mathrm{e}-4$ & 2.141 & $1.247 \mathrm{e}-5$ & 3.970 & 19732.3 & & -88.8 \\
\hline & $T / 160$ & $4.135 e-5$ & 2.083 & $1.919 \mathrm{e}-6$ & 3.982 & 27476.0 & & -86.7 \\
\hline & $T / 200$ & $2.619 \mathrm{e}-5$ & 2.046 & $7.880 \mathrm{e}-7$ & 3.989 & 34849.0 & & -86.2 \\
\hline \multirow{5}{*}{$\begin{array}{l}0 \\
\dot{1} \\
\dot{1} \\
\stackrel{1}{t}\end{array}$} & $T / 50$ & $8.882 \mathrm{e}-6$ & - & $1.374 \mathrm{e}-5$ & 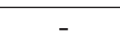 & 15177.6 & \multirow{5}{*}{$\begin{array}{l}\stackrel{0}{1} \\
\stackrel{1}{0}\end{array}$} & -83.4 \\
\hline & $T / 80$ & $1.348 \mathrm{e}-6$ & 3.998 & $2.089 e-6$ & 3.998 & 25255.6 & & -81.8 \\
\hline & $T / 100$ & $5.521 \mathrm{e}-7$ & 3.999 & $8.597 e-7$ & 3.999 & 32653.3 & & -81.8 \\
\hline & $T / 160$ & $8.426 \mathrm{e}-8$ & 4.000 & $1.312 \mathrm{e}-7$ & 4.000 & 58771.3 & & -77.4 \\
\hline & $T / 200$ & $3.451 \mathrm{e}-8$ & 4.000 & $5.375 \mathrm{e}-8$ & 4.000 & 74145.3 & & -66.8 \\
\hline \multirow{5}{*}{ 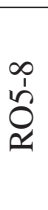 } & $T / 50$ & $1.320 \mathrm{e}-7$ & - & $2.050 \mathrm{e}-7$ & - & 23066.5 & \multirow{5}{*}{$\stackrel{m}{\stackrel{m}{d}}$} & -16.7 \\
\hline & $T / 80$ & $1.263 \mathrm{e}-8$ & 4.992 & $1.965 \mathrm{e}-8$ & 4.990 & 38139.9 & & -5.2 \\
\hline & $T / 100$ & $4.144 \mathrm{e}-9$ & 4.995 & $6.448 \mathrm{e}-9$ & 4.993 & 48088.9 & & -3.8 \\
\hline & $T / 160$ & $3.958 \mathrm{e}-10$ & 4.997 & $6.162 \mathrm{e}-10$ & 4.996 & 79646.1 & & -3.5 \\
\hline & $T / 200$ & $1.297 \mathrm{e}-10$ & 5.000 & $2.021 \mathrm{e}-10$ & 4.997 & 101180.1 & & -3.5 \\
\hline \multirow{5}{*}{$\begin{array}{l}0 \\
1 \\
0 \\
0 \\
\not 1\end{array}$} & $T / 50$ & $1.484 \mathrm{e}-4$ & - & $3.388 \mathrm{e}-7$ & - & 12246.4 & \multirow{5}{*}{$\stackrel{m}{\stackrel{m}{d}}$} & +0.3 \\
\hline & $T / 80$ & $5.739 \mathrm{e}-5$ & 2.021 & $2.058 \mathrm{e}-8$ & 5.960 & 21881.5 & & +7.4 \\
\hline & $T / 100$ & $3.660 \mathrm{e}-5$ & 2.016 & $5.421 \mathrm{e}-9$ & 5.977 & 26139.5 & & -1.4 \\
\hline & $T / 160$ & $1.422 \mathrm{e}-5$ & 2.011 & $3.253 \mathrm{e}-10$ & 5.986 & 47660.7 & & +9.0 \\
\hline & $T / 200$ & $9.085 \mathrm{e}-6$ & 2.008 & $8.550 \mathrm{e}-11$ & 5.988 & 55352.9 & & -2.1 \\
\hline
\end{tabular}



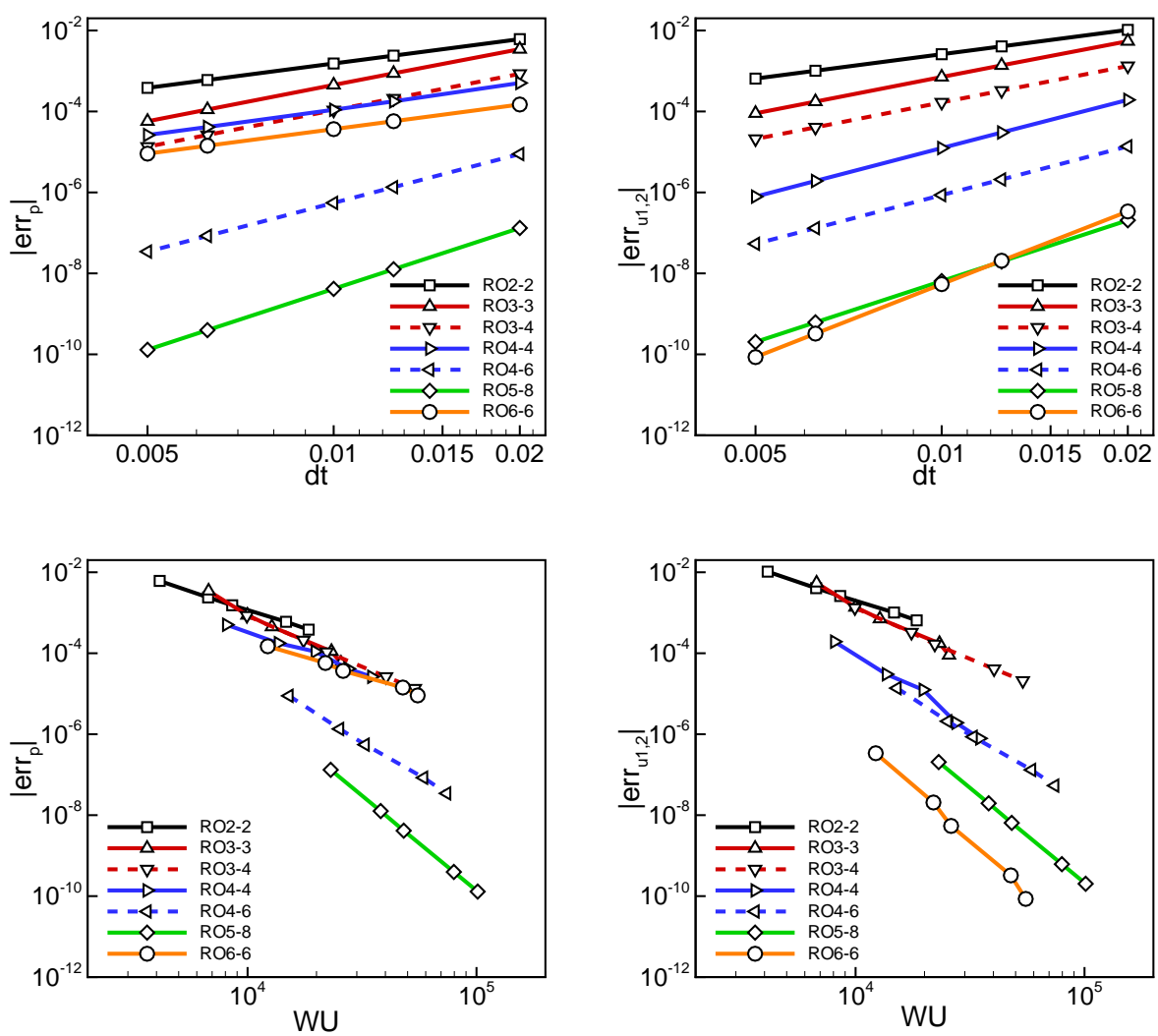

Figure 5: Travelling waves, ROq-s-DG $\mathbb{P}^{6}$ solutions - $L^{2}$ norm of the error with respect to the exact solution vs. time step and Work Units (WU)

airfoil, one of the test cases proposed within the "International Workshop on High-Order CFD Methods", [57]. No subgrid-scale (SGS) model is included in the governing equations, which are simply the equations outlined in Sec. 2, with the DG space discretization and time integration described in Secs. 3.2 and 3.3. The flow around the SD7003 airfoil has been computed for the conditions $\mathrm{M}_{\infty}=0.1, \alpha=8^{\circ}$ and chord-based $\operatorname{Re}_{c}=60000$ on the computational mesh with 20064 50-node hexahedral elements (quartic edge representation) and first cell height $\Delta n / c=$ 0.00029 , corresponding to $\Delta n^{+} \approx 1$, shown in Figure 6(a).

At these flow conditions, a laminar separation bubble (LSB) develops on the suction side of the airfoil, transition takes place in the free shear layer above the airfoil and the flow then reattaches by virtue of the enhanced momentum transport.

Non-reflecting boundary conditions have been imposed at the farfield boundary, located at a distance of $\sim 100 c$ from the wing, no-slip isothermal wall condition with $T_{\text {wall }} / T_{\infty}=1.002$ at the airfoil surface, and periodic boundary conditions over a width $s / c=0.2$ in the spanwise direction.

This test case has been computed by using the highly accurate RO5-8 scheme of Di Marzo, [27], coupled with $\mathbb{P}^{3}$ and $\mathbb{P}^{4}$ DG space approximations, resulting in 401280 DOFs and 702240 


\begin{tabular}{c|c||cccc||ccc}
\hline \hline & \multirow{2}{*}{ DOFs } & \multicolumn{4}{|c||}{ LSB details } & \multicolumn{3}{c}{ Aerodynamic loads } \\
\cline { 3 - 9 } & & $x_{1} / c$ & $x_{2} / c$ & $\mathrm{~L} / c$ & $\mathrm{H} / c$ & $C_{D}$ & $C_{L}$ & $C_{m}$ \\
\hline RO5-8-DG $\mathbb{P}^{3}$ & 401280 & 0.027 & 0.268 & 0.241 & 0.017 & 0.0423 & 0.9615 & -0.0233 \\
RO5-8-DG $P^{4}$ & 702240 & 0.027 & 0.294 & 0.267 & 0.021 & 0.0454 & 0.9534 & -0.0224 \\
\hline DGSEM $\mathbb{P}^{3}[35]$ & $4.26 \mathrm{M}$ & 0.027 & 0.310 & - & - & 0.045 & 0.923 & - \\
DGSEM $\mathbb{P}^{7}[35]$ & $4.55 \mathrm{M}$ & 0.030 & 0.336 & - & - & 0.050 & 0.932 & - \\
\hline Comp. FD O(6) [33] & $53.4 \mathrm{M}$ & 0.031 & 0.303 & 0.272 & 0.020 & 0.0447 & 0.917 & -0.0187 \\
\hline SBP-SAT O(4) $[34]$ & $4.48 \mathrm{M}$ & 0.037 & 0.200 & - & - & 0.034 & 0.968 & - \\
\hline \hline
\end{tabular}

Table 3: SD7003 - Details of the laminar separation bubble and mean aerodynamic loads with number of degrees of freedom per equation (DOFs). $x_{1}$ and $x_{2}$ are the separation and reattachment points coordinates, $L$ and $H$ the separation bubble length and height

DOFs, respectively. The solutions have been advanced in time with a time step equal to a fraction $f=4.4 \mathrm{e}-3$ of the convective time, i.e., $\Delta t=f\left(c / U_{\infty}\right)$, which corresponds to $C F L \approx 15$, where the $C F L$-number has been defined as $C F L=\Delta t U_{\infty} / \Delta n$. The $\mathbb{P}^{3}$ solution has been initialized from a $p$-sequence of lower-order, not fully statistically converged solutions. The mean results shown below have been obtained by averaging both in time and spanwise direction.

For the $\mathbb{P}^{3}$ and $\mathbb{P}^{4}$ solutions, the Figures $6(\mathrm{~b})$ and 7 show the contours of the mean $x$-component of velocity around the airfoil and a detailed view of the flow field and of detachment and reattachment points of the laminar separation bubble. The dimensions of the laminar separation bubble are reported in Table 3 and, along with the mean skin friction and pressure coefficient distributions shown in Figure 9, highlight the noticeable difference of the size of the separation bubble predicted by the $\mathbb{P}^{3}$ and $\mathbb{P}^{4}$ solutions. The mean drag, lift and moment coefficients of the airfoil, are also reported in Table 3 together with results available in the literature.

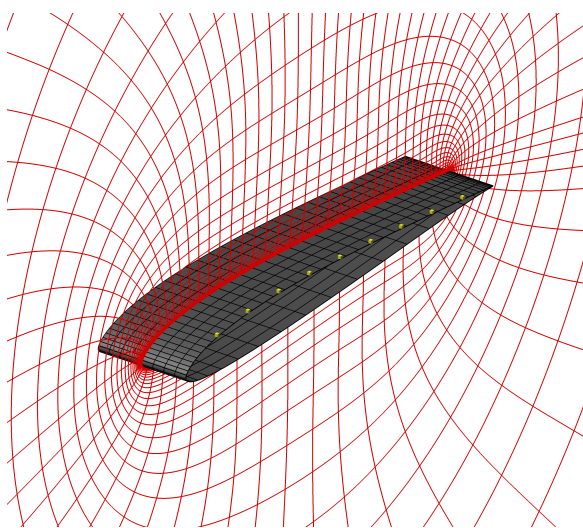

(a) mesh and measurements locations
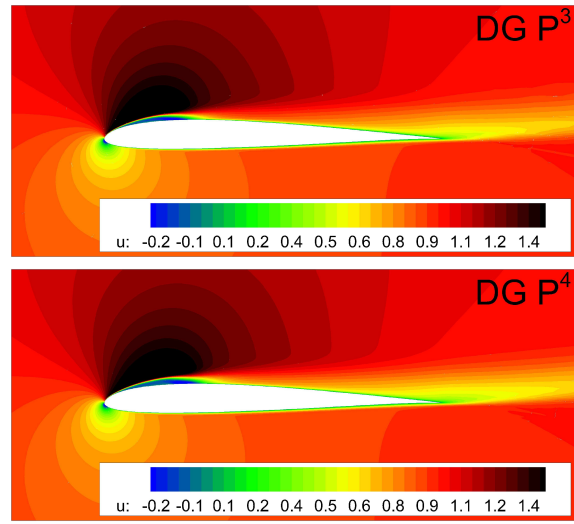

(b) mean $x$-component of velocity

Figure 6: SD7003 - Computational mesh with 20064 50-node hexahedral elements (yellow cubes indicate the chordwise locations of the velocity profiles) and contours of the $x$-component of velocity, RO5-8-DG $\mathbb{P}^{3,4}$ solutions

Figure 8 displays the mean velocity profiles at the chordwise locations indicated in Figure 6(a). The significant difference between the $\mathbb{P}^{3}$ and $\mathbb{P}^{4}$ results clearly requires to assess such results 

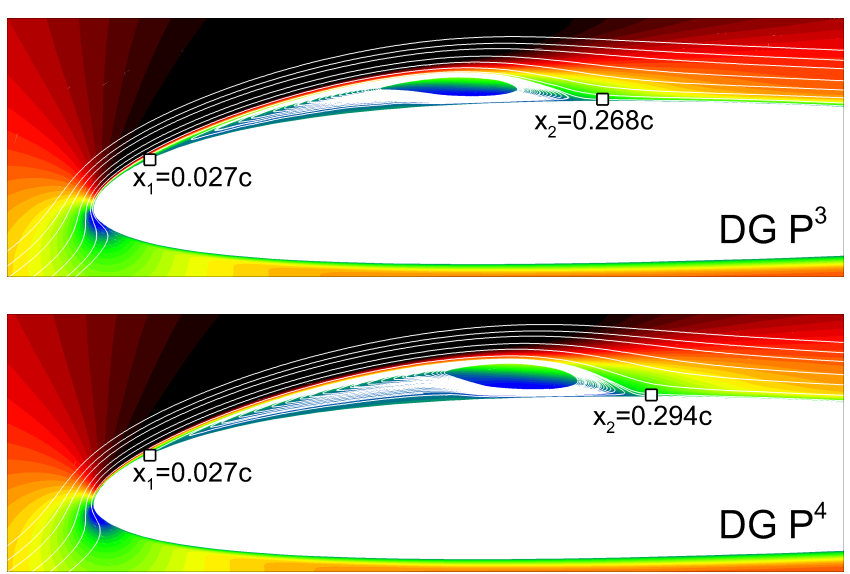

Figure 7: SD7003 - Contours of mean $x$-component of velocity, detail of the laminar separation bubble with indication of the separation and reattachment points, RO5-8-DG $\mathbb{P}^{3,4}$ solutions

against those of more accurate space discretizations, which is the subject of ongoing work. The

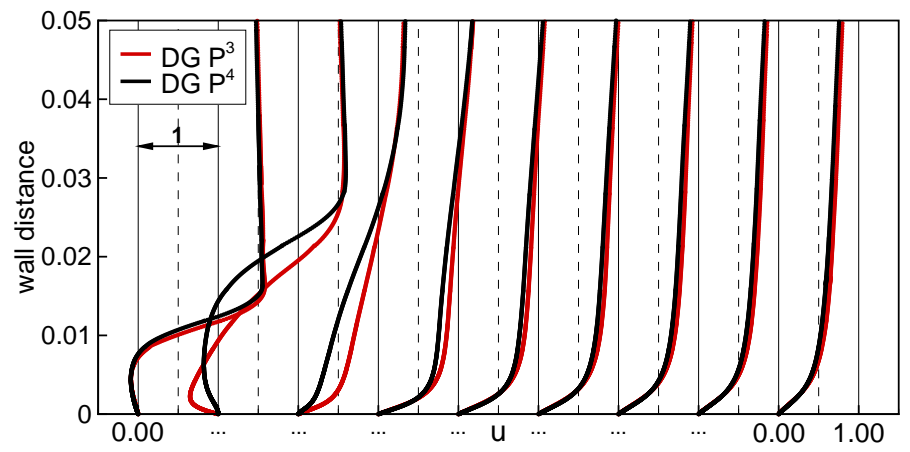

Figure 8: SD7003 - Profiles of mean $x$-component of velocity at chordwise locations $x / c=\{0.1, \ldots, 0.9\}$, RO5-8-DG $\mathbb{P}^{3,4}$ solutions

instantaneous $Q=500$ isosurface of $Q$-criterion for the $\mathbb{P}^{3}$ and $\mathbb{P}^{4}$ solutions is displayed in Figure 10 and shows how a higher-order approximation is able to provide a much better resolution of the vortical structures above the airfoil.

There are several published papers on the LES of this challenging test case, showing a certain dispersion of results due to the complex physical features related to the laminar separation bubble. Unfortunately, no reference DNS database is available for a more reliable comparison. Although it can be expected that our results would improve with $p$ - or $h$-refinement, nevertheless their reasonably good agreement with those reported in [33] and [35] is quite promising.

\section{Conclusions}

In this work we have shown that linearly implicit Rosenbrock-type Runge-Kutta schemes, coupled with a high-order DG space discretization, represent a flexible and effective approach to a globally high-order numerical solution of compressible and incompressible unsteady flows. 

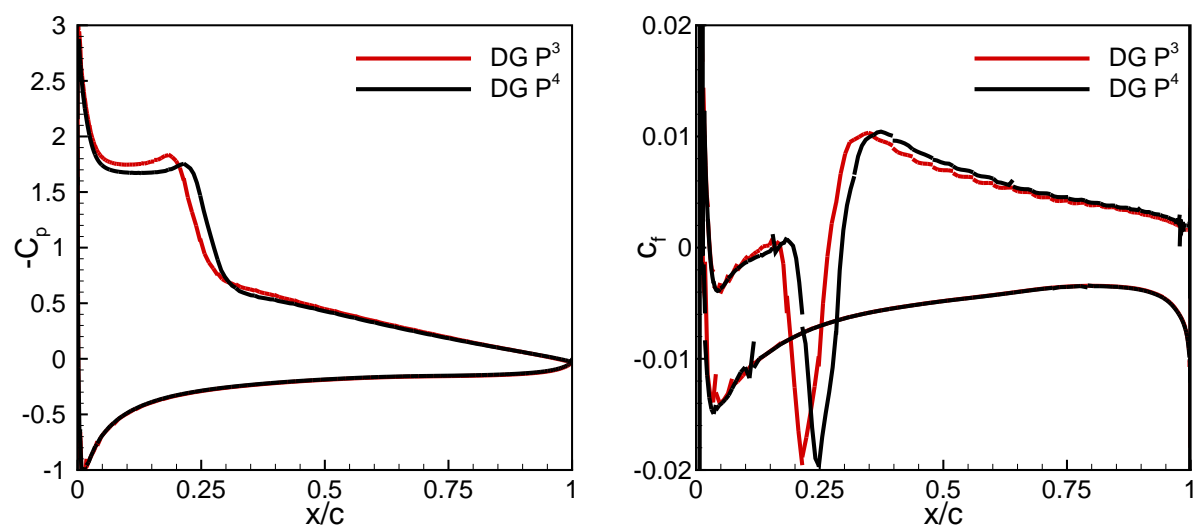

Figure 9: SD7003 - Mean $C_{p}$ and $c_{f}$ distributions, RO5-8-DG $\mathbb{P}^{3,4}$ solutions
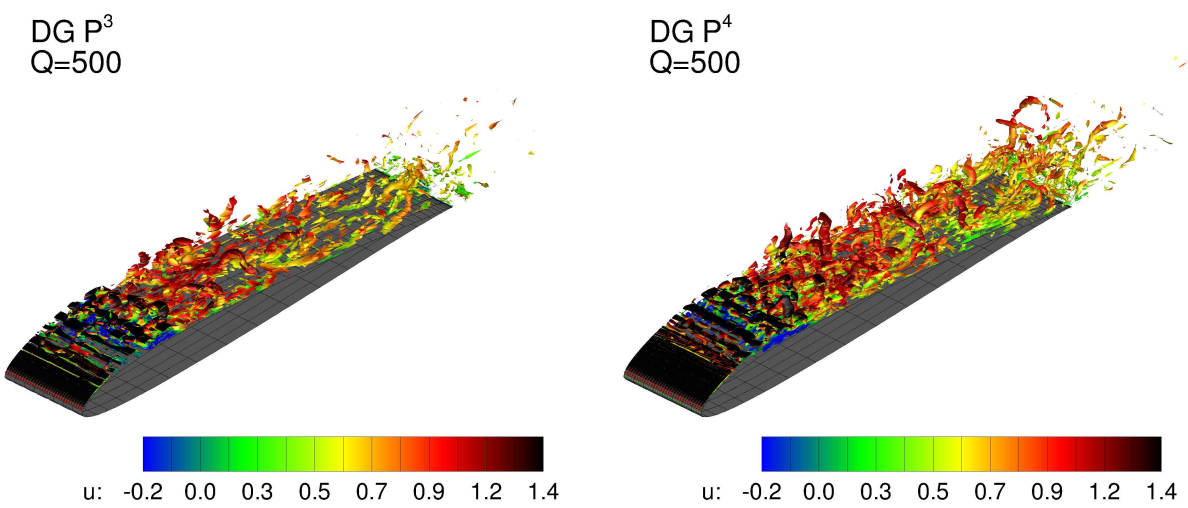

Figure 10: SD7003 - Instantaneous $Q=500$ isosurface of $Q$-criterion, RO5-8-DG $\mathbb{P}^{3,4}$ solutions

It has been proposed an implementation of Rosenbrock schemes for systems of equations with a solution dependent block diagonal matrix multiplying the time derivative, a situation that occurs if a set of primitive variables is chosen for the unknowns in the governing equations of compressible flows. If primitive variables are used as working variables, we have also suggested a simple way to ensure the positivity of all thermodynamic variables at the discrete level.

The assessment of temporal accuracy and performance of several Rosenbrock schemes leads us to conclude that higher-order schemes are much more efficient than lower-order ones if low levels of time integration error are being sought. Applied to the DG ILES of a transitional flow, Rosenbrock schemes provided solutions which are in reasonable good agreement with reference results, proving to be numerically stable and accurate for a time step size significantly larger than that used by other authors in lower order temporal schemes.

Ongoing work is devoted to improve the efficiency of Rosenbrock schemes, by implementing automatic time step adaptation techniques and by increasing the efficiency of iterative solvers, and to a thorough validation of high-order space- and time-accurate DG methods for the simulation of underresolved turbulent flows by means of ILES and hybrid RANS-LES approaches, 
[6].

\section{Acknowledgements}

This work was carried out within the EU FP7 IDIHOM project [58]. HPC resources provided by CINECA within the "Convenzione di Ateneo Università degli Studi di Bergamo" are gratefully acknowledged. The authors would like to thank the reviewers for their valuable comments and suggestions, which helped to improve the manuscript. 


\section{Appendix A. Effect of logarithmic variables on spatial accuracy}

The effect of using logarithmic variables on the accuracy and convergence rate of numerical solutions has been assessed by computing the well-known Ringleb flow problem, see e.g. [59]. In order avoid inaccuracies related to the treatment of slip boundary conditions, which is quite a critical issue for this test case, [60], the exact solution has been set on all the boundaries of the computational domain.

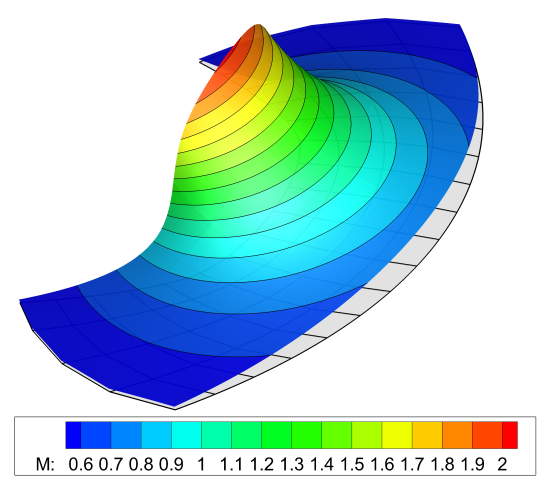

(a) Mach

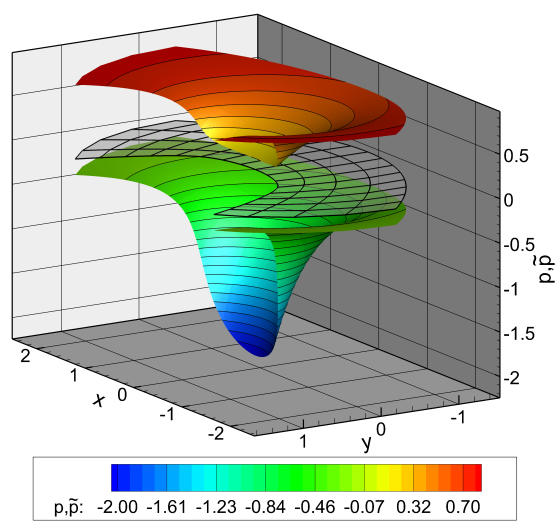

(b) $p$ and $\tilde{p}$

Figure A.11: Ringleb flow - Mach number, pressure, and logarithm of pressure contours, $\mathbb{P}^{6}$ solution on the $16 \times 4$ 17-node quadrilateral elements mesh

Numerical solutions have been computed on a set of eight grids with $16 i \times 4 i, i=1, \cdots, 8,17$ node quadrilateral elements, and up to $\mathbb{P}^{6}$ polynomial approximation, i.e. with $(k+1)(k+2) / 2 \cdot 64 i^{2}$ degrees of freedom (DOFs) per equation. Figure A.11 displays the results of the $\mathbb{P}^{6}$ solution, computed on the $i=1$ grid, represented in terms of Mach number, pressure, and logarithm of pressure contours.

Figure A.12 compares the convergence rate and accuracy of solutions computed using polynomial approximations both for $p$ and $T$ and for their logarithms $\widetilde{p}$ and $\widetilde{T}$. Despite slightly higher $L^{2}$ error norms of the solutions based on polynomial approximations for $\widetilde{p}$ and $\widetilde{T}$, nevertheless the formal order of spatial convergence appears to be equally well verified by both choices of variables. This provides some confidence that using polynomial approximations for $\widetilde{p}$ and $\widetilde{T}$ in place of $p$ and $T$ does not impair the ability of predicting physically correct solutions.

In the authors' experience this approach proved useful to enhance the robustness of high-order simulations of complex transonic flows, as those addressed within the EU FP7 IDIHOM project, see $[61,6]$. Nevertheless, it is not to be intended as a substitute for shock-capturing techniques. 

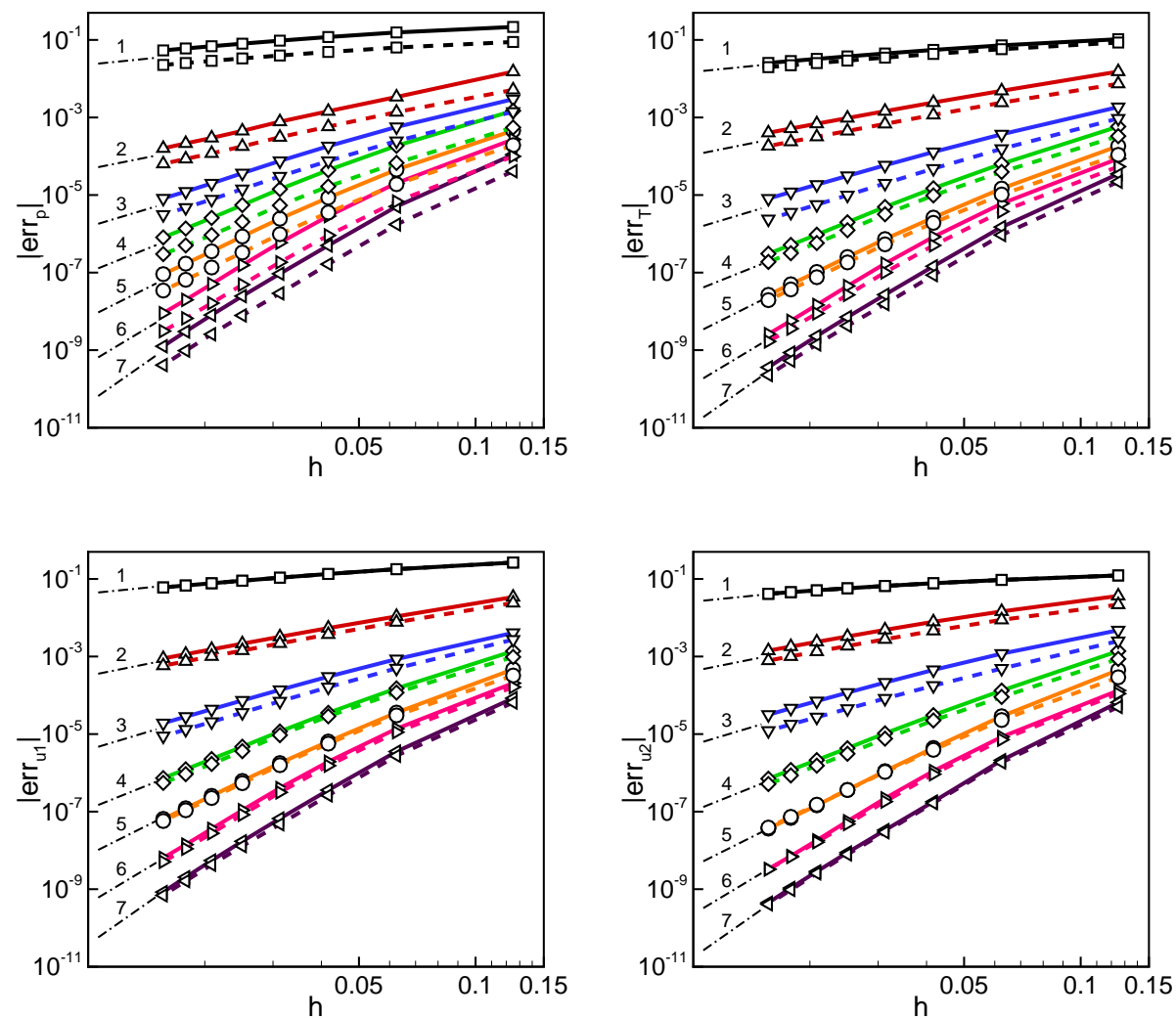

Figure A.12: Ringleb flow $-L^{2}$ error norm on flow variables vs. mesh size. Dashed and solid lines refer to $\mathbf{w}=\left[p, T, u_{i}\right]^{T}$ and to $\mathbf{w}=\left[\widetilde{p}, \widetilde{T}, u_{i}\right]^{T}$ variables, respectively. Dash-dot lines represent the formal order of spatial convergence 
Appendix B. Sets of coefficients of the Rosenbrock schemes considered in this paper

\begin{tabular}{|c|c|c|}
\hline \multicolumn{3}{|c|}{ Iannelli-Baker (RO2-2) [23] } \\
\hline$\gamma$ & $=$ & $1-\frac{\sqrt{2}}{2}$ \\
\hline$a_{21}$ & $=$ & $4-8 \gamma$ \\
\hline$c_{21}$ & $=$ & 0 \\
\hline$m_{1}$ & $=$ & $\frac{1}{\gamma}\left(1-\frac{1}{8 \gamma}\right)$ \\
\hline$m_{2}$ & $=$ & $\frac{1}{8 \gamma^{2}}$ \\
\hline \multicolumn{3}{|c|}{ Lang-Verwer ROS3P (RO3-3) [24] } \\
\hline$\gamma$ & $=$ & $\frac{1}{2}+\frac{\sqrt{3}}{6}$ \\
\hline$a_{21}$ & $=$ & $\gamma^{-1}$ \\
\hline$a_{31}$ & $=$ & $\gamma^{-1}$ \\
\hline$a_{32}$ & $=$ & 0 \\
\hline$c_{21}$ & $=$ & \\
\hline$c_{31}$ & $=$ & $-\gamma^{-1}\left(1+\gamma^{-1}\left(2-\frac{1}{2 \gamma}\right)\right)$ \\
\hline$c_{32}$ & $=$ & $-\gamma^{-1}\left(2-\frac{1}{2 \gamma}\right)$ \\
\hline$m_{1}$ & $=$ & $\gamma^{-1}\left(1+\gamma^{-1}\left(\frac{2}{3}-\frac{1}{6 \gamma}\right)\right)$ \\
\hline$m_{2}$ & $=$ & $\gamma^{-1}\left(\frac{2}{3}-\frac{1}{6 \gamma}\right)$ \\
\hline$m_{3}$ & $=$ & \\
\hline
\end{tabular}

Hairer-Wanner RODAS3 (RO3-4) [25]

\begin{aligned} \hline$\gamma & =1 / 2 \\ a_{21} & =0 \\ a_{31} & =2 \\ a_{32} & =0 \\ a_{41} & =2 \\ a_{42} & =0 \\ a_{43} & =1 \\ c_{21} & =4 \\ c_{31} & =1 \\ c_{32} & =-1 \\ c_{41} & =1 \\ c_{42} & =-1 \\ c_{43} & =-8 / 3 \\ m_{1} & =2 \\ m_{2} & =0 \\ m_{3} & =1 \\ m_{4} & =1\end{aligned}$

Shampine ROS4 (RO4-4) [28]

$$
\begin{aligned}
& \gamma=1 / 2 \\
& a_{21}=2 \\
& a_{31}=48 / 25 \\
& a_{32}=6 / 25 \\
& a_{41}=48 / 25 \\
& a_{42}=6 / 25 \\
& a_{43}=0 \\
& c_{21}=-8 \\
& c_{31}=372 / 25 \\
& c_{32}=12 / 5 \\
& c_{41}=-112 / 125 \\
& c_{42}=-54 / 125 \\
& c_{43}=-2 / 5 \\
& m_{1}=19 / 9 \\
& m_{2}=0.5 \\
& m_{3}=25 / 108 \\
& m_{4}=125 / 108
\end{aligned}
$$

Steinebach RODASP (RO4-6) [26]

\begin{tabular}{rrr}
\hline$\gamma$ & $=$ & $0.25 \mathrm{D}+00$ \\
$a_{21}=$ & $3 \mathrm{D}+00$ \\
$a_{31}=$ & $1.831036793486759 \mathrm{D}+00$ \\
$a_{32}=$ & $4.955183967433795 \mathrm{D}-01$ \\
$a_{41}=$ & $2.304376582692669 \mathrm{D}+00$ \\
$a_{42}=$ & $-5.249275245743001 \mathrm{D}-02$ \\
$a_{43}=$ & $-1.176798761832782 \mathrm{D}+00$ \\
$a_{51}=$ & $-7.170454962423024 \mathrm{D}+00$ \\
$a_{52}=$ & $-4.741636671481785 \mathrm{D}+00$ \\
$a_{53}=$ & $-1.631002631330971 \mathrm{D}+01$ \\
$a_{54}=$ & $-1.062004044111401 \mathrm{D}+00$ \\
$a_{61}=$ & $-7.170454962423024 \mathrm{D}+00$ \\
$a_{62}=$ & $-4.741636671481785 \mathrm{D}+00$ \\
$a_{63}=$ & $-1.631002631330971 \mathrm{D}+01$ \\
$a_{64}=$ & $-1.062004044111401 \mathrm{D}+00$ \\
$a_{65}=$ & $1.0 \mathrm{D}+00$ \\
$c_{21}=$ & $-1.2 \mathrm{D}+01$ \\
$c_{31}=$ & $-8.791795173947035 \mathrm{D}+00$ \\
$c_{32}=$ & $-2.207865586973518 \mathrm{D}+00$ \\
$c_{41}=$ & $1.081793056857153 \mathrm{D}+01$ \\
$c_{42}=$ & $6.780270611428266 \mathrm{D}+00$ \\
& &
\end{tabular}




$$
\begin{array}{rrr}
c_{43}= & 1.953485944642410 \mathrm{D}+01 \\
c_{51}= & 3.419095006749676 \mathrm{D}+01 \\
c_{52}= & 1.549671153725963 \mathrm{D}+01 \\
c_{53}= & 5.474760875964130 \mathrm{D}+01 \\
c_{54}= & 1.416005392148534 \mathrm{D}+01 \\
c_{61}= & 3.462605830930532 \mathrm{D}+01 \\
c_{62}= & 1.530084976114473 \mathrm{D}+01 \\
c_{63}= & 5.699955578662667 \mathrm{D}+01 \\
c_{64}= & 1.840807009793095 \mathrm{D}+01 \\
c_{65}= & -5.714285714285717 \mathrm{D}+00 \\
m_{1}= & -7.170454962423024 \mathrm{D}+00 \\
m_{2}= & -4.741636671481785 \mathrm{D}+00 \\
m_{3}= & -1.631002631330971 \mathrm{D}+01 \\
m_{4}= & -1.062004044111401 \mathrm{D}+00 \\
m_{5}= & 1.0 \mathrm{D}+00 \\
m_{6}= & 1.0 \mathrm{D}+00
\end{array}
$$

Di Marzo, RODAS5-Rod5_1 (RO5-8) [27]

\begin{tabular}{llr}
\hline$\gamma$ & $=$ & $1.9 \mathrm{D}-01$ \\
$a_{21}=$ & $2.0 \mathrm{D}+00$ \\
$a_{31}=$ & $3.040894194418781 \mathrm{D}+00$ \\
$a_{32}=$ & $1.041747909077569 \mathrm{D}+00$ \\
$a_{41}=$ & $2.576417536461461 \mathrm{D}+00$ \\
$a_{42}=$ & $1.622083060776640 \mathrm{D}+00$ \\
$a_{43}=$ & $-9.089668560264532 \mathrm{D}-01$ \\
$a_{51}=$ & $2.760842080225597 \mathrm{D}+00$ \\
$a_{52}=$ & $1.446624659844071 \mathrm{D}+00$ \\
$a_{53}=$ & $-3.036980084553738 \mathrm{D}-01$ \\
$a_{54}=$ & $2.877498600325443 \mathrm{D}-01$ \\
$a_{61}=$ & $-1.409640773051259 \mathrm{D}+01$ \\
$a_{62}=$ & $6.925207756232704 \mathrm{D}+00$ \\
$a_{63}=$ & $-4.147510893210728 \mathrm{D}+01$ \\
$a_{64}=$ & $2.343771018586405 \mathrm{D}+00$ \\
$a_{65}=$ & $2.413215229196062 \mathrm{D}+01$ \\
$a_{71}=$ & $-1.409640773051259 \mathrm{D}+01$ \\
$a_{72}=$ & $6.925207756232704 \mathrm{D}+00$ \\
$a_{73}=$ & $-4.147510893210728 \mathrm{D}+01$ \\
$a_{74}=$ & $2.343771018586405 \mathrm{D}+00$ \\
$a_{75}=$ & $2.413215229196062 \mathrm{D}+01$ \\
$a_{76}=$ & & $1.0 \mathrm{D}+00$ \\
$a_{81}=$ & $-1.409640773051259 \mathrm{D}+01$
\end{tabular}

$$
\begin{aligned}
& a_{82}=6.925207756232704 \mathrm{D}+00 \\
& a_{83}=-4.147510893210728 \mathrm{D}+01 \\
& a_{84}=2.343771018586405 \mathrm{D}+00 \\
& a_{85}=2.413215229196062 \mathrm{D}+01 \\
& \begin{array}{ll}
a_{86}= & 1.0 \mathrm{D}+00
\end{array} \\
& \begin{array}{lll}
a_{87} & = & 1.0 \mathrm{D}+00
\end{array} \\
& c_{21}=-1.031323885133993 \mathrm{D}+01 \\
& c_{31}=-2.104823117650003 \mathrm{D}+01 \\
& c_{32}=-7.234992135176716 \mathrm{D}+00 \\
& c_{41}=3.222751541853323 \mathrm{D}+01 \\
& c_{42}=-4.943732386540191 \mathrm{D}+00 \\
& c_{43}=1.944922031041879 \mathrm{D}+01 \\
& c_{51}=-2.069865579590063 \mathrm{D}+01 \\
& c_{52}=-8.816374604402768 \mathrm{D}+00 \\
& c_{53}=1.260436877740897 \mathrm{D}+00 \\
& c_{54}=-7.495647613787146 \mathrm{D}-01 \\
& c_{61}=-4.622004352711257 \mathrm{D}+01 \\
& c_{62}=-1.749534862857472 \mathrm{D}+01 \\
& c_{63}=-2.896389582892057 \mathrm{D}+02 \\
& c_{64}=9.360855400400906 \mathrm{D}+01 \\
& c_{65}=3.183822534212147 \mathrm{D}+02 \\
& c_{71}=3.420013733472935 \mathrm{D}+01 \\
& c_{72}=-1.415535402717690 \mathrm{D}+01 \\
& c_{73}=5.782335640988400 \mathrm{D}+01 \\
& c_{74}=2.583362985412365 \mathrm{D}+01 \\
& c_{75}=1.408950972071624 \mathrm{D}+00 \\
& c_{76}=-6.551835421242162 \mathrm{D}+00 \\
& c_{81}=4.257076742291101 \mathrm{D}+01 \\
& c_{82}=-1.380770672017997 \mathrm{D}+01 \\
& c_{83}=9.398938432427124 \mathrm{D}+01 \\
& c_{84}=1.877919633714503 \mathrm{D}+01 \\
& c_{85}=-3.158359187223370 \mathrm{D}+01 \\
& c_{86}=-6.685968952921985 \mathrm{D}+00 \\
& c_{87}=-5.810979938412932 \mathrm{D}+00 \\
& m_{1}=-1.409640773051259 \mathrm{D}+01 \\
& m_{2}=6.925207756232704 \mathrm{D}+00 \\
& m_{3}=-4.147510893210728 \mathrm{D}+01 \\
& m_{4}=2.343771018586405 \mathrm{D}+00 \\
& m_{5}=2.413215229196062 \mathrm{D}+01 \\
& m_{6}=1 . \mathrm{D}+00 \\
& m_{7}=1 . \mathrm{D}+00 \\
& \begin{array}{lll}
m_{8}= & 1 . \mathrm{D}+00
\end{array}
\end{aligned}
$$


Kaps and Wanner. ROW6A (RO6-6) [29]

\begin{tabular}{rlr}
\hline$\gamma$ & $=$ & $3.341423670680504 \mathrm{D}-01$ \\
$a_{21}=$ & $2.0 \mathrm{D}+00$ \\
$a_{31}=$ & $1.751493065942685 \mathrm{D}+00$ \\
$a_{32}=$ & $-1.454290536332865 \mathrm{D}-01$ \\
$a_{41}=$ & $-1.847093912231436 \mathrm{D}+00$ \\
$a_{42}=$ & $-2.513756792158473 \mathrm{D}+00$ \\
$a_{43}=$ & $1.874707432337999 \mathrm{D}+00$ \\
$a_{51}=$ & $1.059634783677141 \mathrm{D}+01$ \\
$a_{52}=$ & $1.974951525952609 \mathrm{D}+00$ \\
$a_{53}=$ & $-1.905211286263863 \mathrm{D}+00$ \\
$a_{54}=$ & $-3.575118228830491 \mathrm{D}+00$ \\
$a_{61}=$ & $2.417642067883312 \mathrm{D}+00$ \\
$a_{62}=$ & $3.050984437044573 \mathrm{D}-01$ \\
$a_{63}=$ & $-2.346208879122501 \mathrm{D}-01$ \\
$a_{64}=$ & $-1.327038464607418 \mathrm{D}-01$ \\
$a_{65}=$ & $3.912922779645768 \mathrm{D}-02$ \\
$c_{21}=$ & $-1.745029492512995 \mathrm{D}+01$ \\
$c_{31}=$ & $-1.202359936227844 \mathrm{D}+01$ \\
$c_{32}=$ & $1.315910110742745 \mathrm{D}+00$ \\
$c_{41}=$ & $2.311230597159272 \mathrm{D}+01$ \\
$c_{42}=$ & $1.297893129565445 \mathrm{D}+01$ \\
$c_{43}=$ & $-8.445374594562038 \mathrm{D}+00$ \\
$c_{51}=$ & $-3.147228891330713 \mathrm{D}+00$ \\
$c_{52}=$ & $-1.761332622909965 \mathrm{D}+00$ \\
$c_{53}=$ & $6.115295934038585 \mathrm{D}+00$ \\
$c_{51}=$ & $1.499319950457112 \mathrm{D}+01$ \\
$c_{62}=$ & $-2.015840911262880 \mathrm{D}+01$ \\
$c_{63}=$ & $-1.603923799800133 \mathrm{D}+00$ \\
$c_{64}=$ & $1.155870096920252 \mathrm{D}+00$ \\
$c_{65}=$ & $6.304639815292044 \mathrm{D}-01$ \\
$c_{66}=$ & $-1.602510215637174 \mathrm{D}-01$ \\
$m_{1}=$ & $3.399347452674165 \mathrm{D}+01$ \\
$m_{2}=$ & $-2.091829882847333 \mathrm{D}+01$ \\
$m_{3}=$ & $-1.375688477471081 \mathrm{D}+01$ \\
$m_{4}=$ & $-1.113925929930077 \mathrm{D}+01$ \\
$m_{5}=$ & $2.873406527609468 \mathrm{D}+00$ \\
$m_{6}=$ & $3.876609945620840 \mathrm{D}+01$
\end{tabular}




\section{Appendix C. Stability of Rosenbrock schemes: $d_{i j}$ parameters and $G^{(s)}$ matrices}

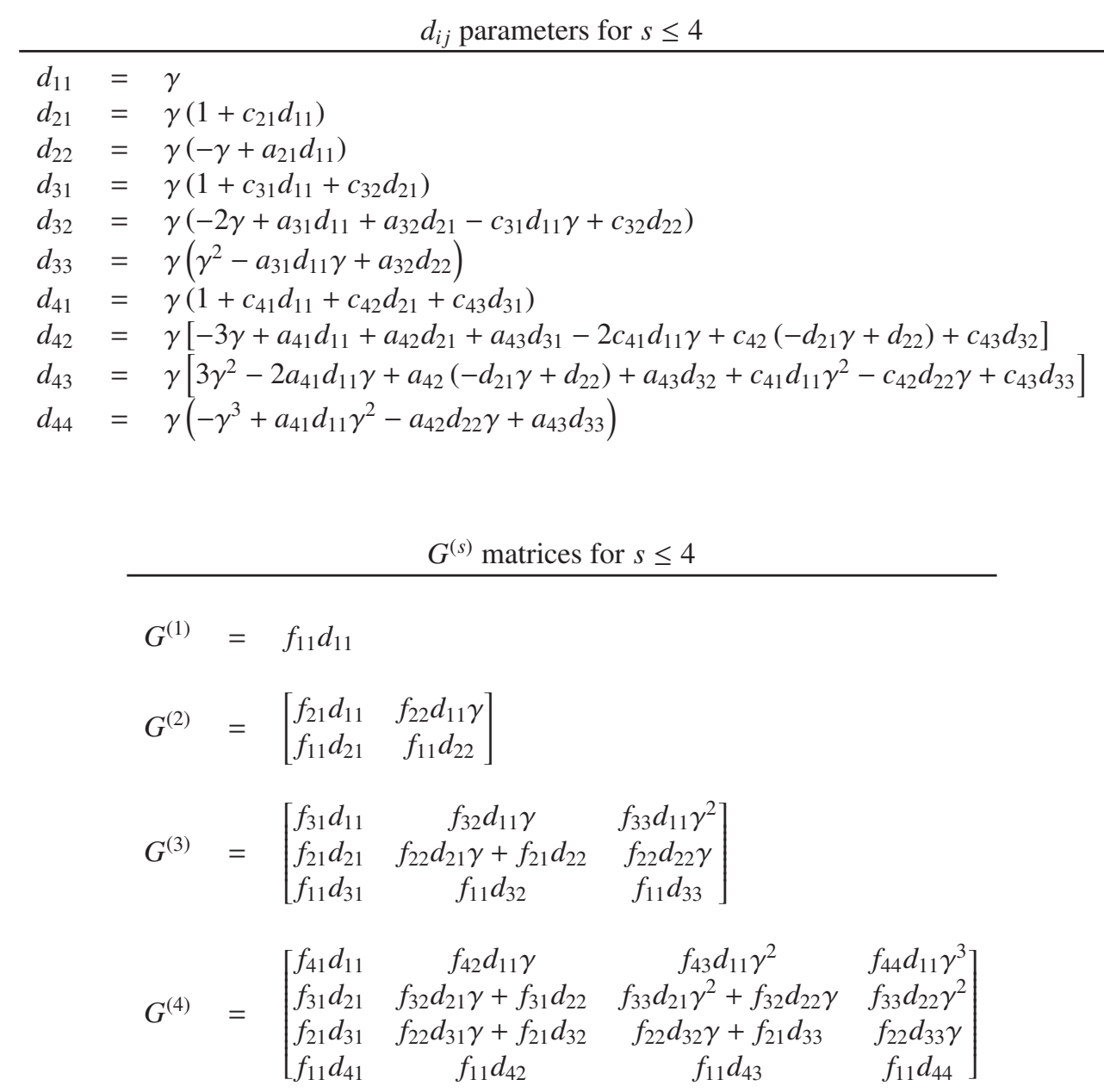

[1] J.-B. Chapelier, M. de la Llave Plata, F. Renac, E. Lamballais, Evaluation of a high-order discontinuous Galerkin method for the DNS of turbulent flows, Comput. Fluids 95 (0) (2014) 210 - 226. doi:http://dx.doi.org/10.1016/j.compfluid.2014.02.015.

[2] C. Carton de Wiart, K. Hillewaert, M. Duponcheel, G. Winckelmans, Assessment of a discontinuous Galerkin method for the simulation of vortical flows at high Reynolds number, Int. J. Numer. Methods Fluids 74 (7) (2014) 469-493. doi:10.1002/fld.3859.

[3] L. Wei, A. Pollard, Direct numerical simulation of compressible turbulent channel flows using the discontinuous Galerkin method, Comput. Fluids 47 (1) (2011) 85 - 100. doi:http://dx.doi.org/10.1016/j.compfluid.2011.02.015.

[4] F. van der Bos, B. J. Geurts, Computational error-analysis of a discontinuous Galerkin discretization applied to large-eddy simulation of homogeneous turbulence, Computer Methods in Applied Mechanics and Engineering 199 (1316) (2010) 903 - 915. doi:http://dx.doi.org/10.1016/j.cma.2009.08.026.

[5] A. Uranga, P.-O. Persson, M. Drela, J. Peraire, Implicit Large Eddy Simulation of transition to turbulence at low Reynolds numbers using a Discontinuous Galerkin method, International Journal for Numerical Methods in Engineering 87 (1-5) (2011) 232-261. doi:10.1002/nme.3036.

[6] F. Bassi, L. Botti, A. Colombo, A. Crivellini, A. Ghidoni, A. Nigro, S. Rebay, Time Integration in the Discontinuous Galerkin Code MIGALE - Unsteady Problems, in: N. Kroll, C. Hirsch, F. Bassi, C. Johnston, K. Hillewaert (Eds.), 
IDIHOM: Industrialization of High-Order Methods - A Top-Down Approach, Vol. 128 of Notes on Numerical Fluid Mechanics and Multidisciplinary Design, Springer International Publishing, 2015, pp. 205-230. doi:10.1007/9783-319-12886-3 11.

URL http://dx.doi.org/10.1007/978-3-319-12886-3_11

[7] P. Spalart, W. Jou, M. Strelets, S. Allmaras, Comments on the feasibility of LES for wings, and on a hybrid RANS/LES approach, in: International Conference on DNS/LES, Aug. 4-8, 1997, Ruston, Louisiana., 1997, pp. $137-147$.

[8] M. Strelets, Detached eddy simulation of massively separated flows, American Institute of Aeronautics and Astronautics, 2001. doi:doi:10.2514/6.2001-879. URL http://dx.doi.org/10.2514/6.2001-879

[9] J. C. Kok, H. S. Dol, B. Oskam, H. van der Ven, Extra-large eddy simulation of massively separated flows, AIAA Paper 2004-264 (2004).

[10] G. Gassner, M. Dumbser, F. Hindenlang, C.-D. Munz, Explicit one-step time discretizations for discontinuous Galerkin and finite volume schemes based on local predictors, J. Comput. Phys. 230 (11) (2011) 4232 - 4247, special issue High Order Methods for CFD Problems.

[11] F. Hindenlang, G. Gassner, C. Altmann, A. Beck, M. Staudenmaier, C.-D. Munz, Explicit Discontinuous Galerkin methods for unsteady problems, Comput. Fluids 61 (2012) 86-93. URL http://dx.doi.org/10.1016/j.compfluid.2012.03.006

[12] P.-O. Persson, High-Order LES Simulations using Implicit-Explicit Runge-Kutta Schemes, American Institute of Aeronautics and Astronautics, 2011. doi:doi:10.2514/6.2011-684. URL http://dx.doi .org/10.2514/6.2011-684

[13] C. F. Curtiss, J. O. Hirschfelder, Integration of stiff equation, in: Proc. Natl. Acad. Sci. USA, 1952, pp. $235-243$.

[14] C. A. Kennedy, M. H. Carpenter, Additive Runge-Kutta Schemes for Convection-Diffusion-Reaction equations, Technical Memorandum TM-2001-211038, NASA (July 2001).

[15] M. H. Carpenter, C. A. Kennedy, H. Bijl, S. A. Viken, V. N. Vatsa, Fourth-order Runge-Kutta schemes for fluid mechanics applications, J. Sci. Comput. 25 (1) (2005) 157-194.

[16] J. R. Cash, On the integration of stiff systems of O.D.E.s using Extended Backward Differentiation Formulae, Numerische Mathematik (34) (1980) 235-246.

[17] J. R. Cash, The integration of stiff initial value problems in ODEs using Modified Extended Backward Differentiation Formulae, Computers \& Mathematics with Applications 5 (9) (1983) 645-657.

[18] A. Nigro, A. Ghidoni, S. Rebay, F. Bassi, Modified Extended BDF scheme for the discontinuous Galerkin solution of unsteady compressible flows, Int. J. Numer. Methods Fluids 76 (9) (2014) 549-574. doi:10.1002/fld.3944. URL http://dx.doi.org/10.1002/fld. 3944

[19] G.-Y. Psihoyios, J. Cash, A stability result for general linear methods with characteristic function having real poles only, BIT Numerical Mathematics 38 (3) (1998) 612-617. doi:10.1007/BF02510263.

[20] A. Nigro, C. De Bartolo, F. Bassi, A. Ghidoni, Up to sixth-order accurate A-stable implicit schemes applied to the discontinuous Galerkin discretized Navier-Stokes equations, J. Comput. Phys. 276 (0) (2014) 136 - 162.

[21] E. Hairer, G. Wanner, Solving ordinary differential equations II, Stiff and differential-algebraic problems, Springer Verlag, 2002

[22] F. Bassi, A. Colombo, C. De Bartolo, N. Franchina, A. Ghidoni, A. Nigro, Investigation of high-order temporal schemes for the discontinuous Galerkin solution of the Navier-Stokes equations, 11th World Congress on Computational Mechanics, WCCM 2014, 5th European Conference on Computational Mechanics, ECCM 2014 and 6th European Conference on Computational Fluid Dynamics, ECFD 2014 (2014) 5651-5662.

[23] G. S. Iannelli, A. J. Baker, A stiffly-stable implicit Runge-Kutta algorithm for CFD applications, AIAA Paper 88-0416, AIAA (1988)

[24] J. Lang, J. Verwer, ROS3P-An accurate third-order Rosenbrock solver designed for parabolic problems, BIT 41 (4) (2001) 731-738.

[25] E. Hairer, G. Wanner, Solving Ordinary Differential Equations II, Stiff and Differential-Algebraic Problems (2nd revised edition), Springer Series in Computational Mathematics, 1996.

[26] G. Steinebach, Order-reduction of ROW-methods for DAEs and method of lines applications, Preprint-Nr. 1741, FB Mathematik, TH Darmstadt (1995).

[27] G. Di Marzo, RODAS5(4) - Méthodes de Rosenbrock d'ordre 5(4) adaptées aux problemes différentielsalgébriques, MSc Mathematics Thesis; Faculty of Science, University of Geneva, Switzerland.

[28] W. H. Press, S. A. Teukolsky, W. T. Vetterling, B. P. Flannery, Numerical Recipes in FORTRAN; The Art of Scientific Computing, 2nd Edition, Cambridge University Press, New York, NY, USA, 1993.

[29] P. Kaps, G. Wanner, A Study of Rosenbrock-Type Methods of High Order, Numerische Mathematik (38) (1981) 279-298

[30] Y. Zhou, Z. J. Wang, Implicit Large Eddy Simulation of Transitional Flow over a SD7003 Wing Using High-order Spectral Difference Method, AIAA Paper 2010-4442 (2010). 
[31] P. Castonguay, C. Liang, A. Jameson, Simulation of Transitional Flow over Airfoils using the Spectral Difference Method, AIAA Paper 2010-4626 (2010).

[32] C. Carton de Wiart, K. Hillewaert, DNS and ILES of transitional flows around a SD7003 using a high order Discontinuous Galerkin Method, Seventh International Conference on Computational Fluid Dynamics (ICCFD7) ICCFD7-3604 (2012).

[33] D. J. Garmann, M. R. Visbal, C3.3: Implicit large eddy-simulations of transitional flow over the SD7003 airfoil using compact finite-differencing and filtering, in: 2nd International Workshop on High-Order CFD Methods, Cologne, Germany, 2013, http://www.dlr.de/as/Portaldata/5/Resources/dokumente/veranstaltungen/2013_05hiocfd/contrib/C33_Garmann.pdf.

[34] P. D. Boom, D. W. Zingg, Time-accurate flow simulations using an efficient Newton-Krylov-Schur approach with high-order temporal and spatial discretization, AIAA Paper 2012-0383 (2013).

[35] T. Bolemann, A. Beck, D. Flad, H. Frank, V. Mayer, C.-D. Munz, High-Order Discontinuous Galerkin Schemes for Large-Eddy Simulations of Moderate Reynolds Number Flows, in: N. Kroll, C. Hirsch, F. Bassi, C. Johnston, K. Hillewaert (Eds.), IDIHOM: Industrialization of High-Order Methods - A Top-Down Approach, Vol. 128 of Notes on Numerical Fluid Mechanics and Multidisciplinary Design, Springer International Publishing, 2015, pp. 435-456. doi:10.1007/978-3-319-12886-3_20.

URL http://dx.doi .org/10.1007/978-3-319-12886-3_20

[36] F. Bassi, S. Rebay, G. Mariotti, S. Pedinotti, M. Savini, A high-order accurate discontinuous finite element method for inviscid and viscous turbomachinery flows, in: R. Decuypere, G. Dibelius (Eds.), Proceedings of the 2nd European Conference on Turbomachinery Fluid Dynamics and Thermodynamics, Technologisch Instituut, Antwerpen, Belgium, 1997, pp. 99-108.

[37] F. Bassi, A. Crivellini, D. A. Di Pietro, S. Rebay, An artificial compressibility flux for the discontinuous Galerkin solution of the incompressible Navier-Stokes equations, J. Comput. Phys. 218 (2006) 794-815.

[38] F. Bassi, S. Rebay, A high order discontinuous Galerkin method for compressible turbulent flows, in: Discontinuous Galerkin Methods. Theory, Computation and Applications, Vol. 11 of Lecture Notes in Computational Science and Engeneering, Springer-Verlag, 2000, pp. 77-88, First Internation Symposium on Discontinuous Galerkin Methods, May 24-26, 1999, Newport, RI, USA

[39] V. Dolejší, Semi-implicit interior penalty discontinuous Galerkin methods for viscous compressible flows, Commun. Comput. Phys. 4 (2008) 231-274.

[40] G. J. Gassner, F. Lörcher, C.-D. Munz, A discontinuous Galerkin scheme based on a space-time expansion II. Viscous flow equations in multi dimensions, J. Sci. Comput. 34 (2008) 260-286.

[41] L. Botti, Influence of reference-to-physical frame mappings on approximation properties of discontinuous piecewise polynomial spaces, J. Sci. Comput. (2011) 1-29.

[42] P. Tesini, An h-Multigrid Approach for High-Order Discontinuous Galerkin Methods, Ph.D. thesis, University of Bergamo, Bergamo, Italy (January 2008).

[43] F. Bassi, L. Botti, A. Colombo, D. A. Di Pietro, P. Tesini, On the flexibility of agglomeration based physical space discontinuous Galerkin discretizations, J. Comput. Phys. 231 (1) (2012) 45 - 65. doi:10.1016/j.jcp.2011.08.018.

[44] D. A. Di Pietro, A. Ern, Mathematical Aspects of Discontinuous Galerkin Methods, Vol. 69 of Mathématiques et Applications, Springer-Verlag, 2012.

[45] Y.-H. Choi, C. L. Merkle, The application of preconditioning in viscous flows, J. Comput. Phys. 105 (2) (1993) 207 - 223. doi:http://dx.doi.org/10.1006/jcph.1993.1069.

[46] J. M. Weiss, W. A. Smith, Preconditioning applied to variable and constant density flows, AIAA J. 33 (11) (1995) 2050-2057.

[47] F. Bassi, C. De Bartolo, R. Hartmann, A. Nigro, A discontinuous Galerkin method for inviscid low Mach number flows, J. Comput. Phys. 228 (11) (2009) 3996 - 4011. doi:http://dx.doi.org/10.1016/j.jcp.2009.02.021.

[48] F. Brezzi, G. Manzini, D. Marini, P. Pietra, A. Russo, Discontinuous Galerkin approximations for elliptic problems, Numer. Meth. Part. D. E. 16 (2000) 365-378.

[49] D. N. Arnold, F. Brezzi, B. Cockburn, L. D. Marini, Unified analysis of discontinuous Galerkin methods for elliptic problems, SIAM J. Numer. Anal. 39 (5) (2002) 1749-1779.

[50] J. J. Gottlieb, C. P. T. Groth, Assessment of Riemann solvers for unsteady one-dimensional inviscid flows of perfect gases, J. Comput. Phys. 78 (1988) 437-458.

[51] D. Hänel, R. Schwane, G. Seider, On the accuracy of upwind schemes for the solution of the Navier-Stokes equations, AIAA Paper 87-1105 (1987).

[52] A. Crivellini, V. D’Alessandro, F. Bassi, A Spalart-Allmaras turbulence model implementation in a discontinuous Galerkin solver for incompressible flows, J. Comput. Phys. 241 (2013) 388-415. doi:http://dx.doi.org/10.1016/j.jcp.2012.12.038.

[53] F. Bassi, A. Crivellini, D. A. Di Pietro, S. Rebay, An implicit high-order discontinuous Galerkin method for steady and unsteady incompressible flows, Comput. Fluids 36 (2007) 1529-1546.

[54] S. Balay, M. F. Adams, J. Brown, P. Brune, K. Buschelman, V. Eijkhout, W. D. Gropp, K. D., M. G. Knepley, L. C. 
McInnes, F. Barry, K. R. Smith, H. Zhang, PETSc Web page, http://www .mcs .anl .gov/petsc (2014).

URL http://www.mcs.anl.gov/petsc

[55] G. S. Iannelli, A. J. Baker, A stiffly-stable implicit Runge-Kutta algorithm for CFD applications, AIAA Paper 88-0416 (1988).

[56] C. Hu, C.-W. Shu, Weighted essentially non-oscillatory schemes on triangular meshes, J. Comput. Phys. 150 (1) (1999) 97-127. doi:http://dx.doi.org/10.1006/jcph.1998.6165.

[57] Z. J. Wang, K. Fidkowski, R. Abgrall, F. Bassi, D. Caraeni, A. Cary, H. Deconinck, R. Hartmann, K. Hillewaert, H. T. Huynh, N. Kroll, G. May, P.-O. Persson, B. van Leer, M. Visbal, High-order CFD methods: Current status and perspective, Int. J. Numer. Methods Fluids 72 (8) (2013) 811-845.

[58] IDIHOM, Industrialisation of High-Order Methods - A top-down approach, Specific Targeted Research Project supported by European Commission, URL: http://www.dlr.de/as/en/desktopdefault.aspx/tabid-7027/11654_read$27492 /$.

[59] G. Chiocchia, Exact solutions to transonic and superesonic flows, Tech. Rep. AGARD-AR-211, Center for Aerospace Information, NASA (1985)

[60] F. Bassi, S. Rebay, High-order accurate discontinuous finite element solution of the 2D Euler equations, J. Comput. Phys. 138 (1997) 251-285.

[61] F. Bassi, L. Botti, A. Colombo, A. Crivellini, C. De Bartolo, N. Franchina, A. Ghidoni, S. Rebay, Time Integration in the Discontinuous Galerkin code MIGALE - Steady Problems, in: N. Kroll, C. Hirsch, F. Bassi, C. Johnston, K. Hillewaert (Eds.), IDIHOM: Industrialization of High-Order Methods - A Top-Down Approach, Vol. 128 of Notes on Numerical Fluid Mechanics and Multidisciplinary Design, Springer International Publishing, 2015, pp. 179-204. doi:10.1007/978-3-319-12886-3_10.

URL http://dx.doi .org/10.1007/978-3-319-12886-3_10 